TUW-96-27 and gr-qc/9612021

\title{
Boundary Conditions and Quasilocal Energy in the Canonical Formulation of All $1+1$ Models of Gravity*
}

\author{
W. Kummer and S. R. Lau ${ }^{\dagger}$ \\ Institut für Theoretische Physik, \\ Technische Universität Wien, \\ Wiedner Hauptstraße 8-10, \\ A-1040 Wien, Österreich
}

\begin{abstract}
Within a first-order framework, we comprehensively examine the role played by boundary conditions in the canonical formulation of a completely general two-dimensional gravity model. Our analysis particularly elucidates the perennial themes of mass and energy. The gravity models for which our arguments are valid include theories with dynamical torsion and so-called generalized dilaton theories (GDTs). Our analysis of the canonical action principle (i) provides a rigorous correspondence between the most general first-order two-dimensional Einstein-Cartan model (ECM) and GDT and (ii) allows us to extract in a virtually simultaneous manner the "true degrees of freedom" for both ECMs and GDTs. For all such models, the existence of an absolutely conserved (in vacuo) quantity $C$ is a generic feature, with (minus) $C$ corresponding to the black-hole mass parameter in the important special cases of spherically symmetric four-dimensional general relativity and standard two-dimensional dilaton gravity. The mass $C$ also includes (minimally coupled) matter into a "universal mass function." We place particular emphasis on the (quite general) class of models within GDT possessing a Minkowski-like groundstate solution (allowing comparison between $C$ and the Arnowitt-Deser-Misner mass for such models).
\end{abstract}

Vienna, December 1996

*Research supported by the "Fonds zur Förderung der wissenschaftlichen Forschung" in Austria.

${ }^{\dagger}$ email addresses: wkummer@tph.tuwien.ac.at and lau@tph16.tuwien.ac.at 


\section{INTRODUCTION AND PRELIMINARIES}

That black-hole physics plays a basic role in understanding the relationships between quantum-mechanics, thermodynamics, and gravity is, by now, undisputed. It is also widely believed that the "correct" notion of gravitational (plus matter) energy is one basic stepping stone towards a deeper understanding of such interconnections. Now, in fact, a plethora of interesting and useful definitions of gravitational "energy" and "mass" exist [1]. Although many of these proposals are quite general, in practice the first test-solutions for which a given proposal is evaluated are the spherically symmetric Schwarzschild and Reissner-Nordström black holes (SBH and RNBH). Therefore, "spherically symmetric general relativity" (SSGR) continues to provide an important arena for examining the notion of gravitational energy. SSGR is effectively a two-dimensional theory [2 4], as the angular coordinates can often be "thrown away," leaving the remaining time and radial dimensions $(t, r)$ as the only coordinates of importance. In this regard SSGR is similar to a generic 1+1-dimensional covariant gravity "model" [5 8], which suggests that there are in effect many "testing grounds" where one can gain experience and insight (which might be brought to bear later on the above mentioned issues). Along this line of research, it would seem quite important not to limit the collection of $1+1$ models a priori, as particular models may not mimic certain important features of SSGR. In particular, the currently popular two-dimensional dilaton gravity (2dDG) 9 11] possesses a dilaton black-hole (DBH) solution which differs physically from the SBH in a potentially severe way [12].

Comparing with such grand intentions, we should point out that the purpose of this paper is more modest. Within a first-order framework, we comprehensively examine the role played by boundary conditions in the canonical formulation of a completely general two-dimensional gravity model. Our analysis particularly elucidates the perennial themes of mass and energy. The definition of energy that we use corresponds to the one [13] derived for full general relativity by Brown and York via a Hamilton-Jacobi-type analysis of the gravitational action, while the mass definition we study corresponds to a plethora of equivalent mass definitions in SSGR [1, 3, 4, 14, 15]. The gravity models for which our arguments are valid include theories with dynamical torsion and so-called generalized dilaton theories (GDTs) [16]. Our analysis of the canonical action (i) provides a rigorous correspondence between the most general 2d Einstein-Cartan model (ECM) and GDT and (ii) allows us to extract in a virtually simultaneous manner the true degrees of freedom for both the (vacuum) ECMs and GDTs we study. For all such models, the existence of an absolutely conserved (in vacuo) quantity $C$ is a generic feature, with (minus) $C$ corresponding to the black-hole mass parameter in the important special cases of spherically symmetric four-dimensional general relativity SSGR and 2dDG [7]. The mass $C$ also includes (minimally coupled) matter into a "universal mass function." (While we do not make a detailed examination of a particular coupled gravitymatter system in this paper, many of our results are unaffected by the inclusion of minimally

\footnotetext{
${ }^{1}$ Thereby complementing the covariant argument [8] which establishes this equivalence. However, our approach is more rigorous as we pay strict attention to the important role played by boundary conditions in the equivalence.
} 
coupled matter. Throughout the course of our discussion, we point out precisely when and why we assume the vacuum case.) We place particular emphasis on the (quite general) class of models within GDT possessing a Minkowski-like groundstate solution [allowing comparison between $C$ and the Arnowitt-Deser-Misner (ADM) mass [17 for such models].

In what remains of this first section we collect our basic notations and geometric conventions, as well as summarize some essential material on which the present work is based. In $\S$ II we discuss the kinematic relationship between certain ADM decompositions of spacetime and two-dimensional moving frames (zweibeine). In $\S$ III we consider the general solution for the spacetime metric corresponding to the models we study, while in $\S$ IV we focus attention on those models which we consider to be "physical". We carry out our investigation of gravitational energy and mass in the central $\S \mathrm{V}$, and the results of this section are applied in $\S$ VI. Finally, $\S$ VII offers some concluding remarks and outlook.

\section{A. Geometry of spacetime $\mathcal{M}$}

Consider a spacetime region $\mathcal{M}$ with boundary $\partial \mathcal{M}$. We put off a detailed description of $\mathcal{M}$ 's boundary structure until the next section. For the moment, it suffices to note that $\partial \mathcal{M}$ is composed of both timelike and spacelike elements. On $\mathcal{M}$ we have a zweibein $e^{a}{ }_{\mu}$, with corresponding Lorentz-signature metric $g_{\mu \nu}$. Coordinate indices $(\mu, \nu, \cdots)$ run over $(t, r)$, zweibein indices $(a, b, \cdots)$ run over $(+,-)$, and the frame or "internal" metric is $\eta_{a b}$ (with $\eta_{+-}=-1$ and $\eta_{++}=\eta_{--}=0$ ). Let $\omega^{a}{ }_{b \mu}=:-\epsilon_{b}^{a} \omega_{\mu}$ denote the coefficients (with respect to the zweibein) of the spacetime connection which, though compatible with the frame metric $\eta_{a b}$, is not necessarily torsion-free. Therefore, in general, $\omega^{a}{ }_{b \mu}$ is built both from the zweibein $e^{a}{ }_{\mu}$ and the spacetime torsion tensor $T^{\lambda}{ }_{\mu \nu}$ (skew in its last two indices). We let $D_{\mu}$ represent the associated covariant derivative operator which "sees" zweibein indices, reserving $\nabla_{\mu}$ for the standard coordinate covariant derivative. Finally, the $\mathcal{M}$ permutation symbol is fixed by $\epsilon_{+-}=-1$, and the corresponding volume form is $\epsilon=\frac{1}{2} \epsilon_{a b} e^{a} \wedge e^{b}$.

\section{B. Action for an Einstein-Cartan model}

The action functional $L$ which describes our spacetime geometry is the one appropriate for a general ECM [5 [7], namely, a first-order action in terms of (what turn out to be) Cartan variables, $]^{2}$

$$
L=\kappa \int_{\mathcal{M}}\left(-X_{a} D e^{a}+X \mathrm{~d} \omega-V \epsilon\right)+L_{\partial \mathcal{M}}+L^{(m)}-\left.L\right|^{0},
$$

\footnotetext{
${ }^{2}$ We remark that the first-order ECM action is an example of a Poisson-sigma model, although as we have written (1.1), the underlying Poisson structure is not manifest as it is in Refs. [5, 6. Our action is far from the most general Poisson-sigma model. Nevertheless, it suffices for our general investigation of $1+1$ gravity models. For a detailed treatment of and unifying approach for $1+1$ gravity based on the Poisson-sigma-model formulation, see Refs. [6] by Klösch and Strobl.
} 
where for convenience ${ }^{\text {f }}$ we have switched to the index-free language of differential forms to write (1.1). Let us provide a brief description of the various terms found in (1.1). First, $L^{(m)}$ represents a possible matter contribution to the action, and $\left.L\right|^{0}$ represents a possible reference term (an essentially arbitrary functional of boundary data which is fixed in the variational principle) [18,13. Such a reference term does not affect the variational principle. For simplicity we shall ignore the reference term until $\S \mathrm{VI}$, when its presence is important for deriving the correct concept of "rest-frame energy." $L_{\partial \mathcal{M}}$ represents a boundary term which is included in the definition of $L$. In this section, we shall assume that

$$
L_{\partial \mathcal{M}}=-\kappa \oint_{\partial \mathcal{M}} X \omega
$$

although later in $\S \mathrm{V}$ we find the need to introduce an "improved" (zweibein-gauge independent) version of this integral. Via an integration by parts we can replace the term $X \mathrm{~d} \omega$ in the volume integral with $\omega \wedge \mathrm{d} X$, along the way generating a boundary term which exactly cancels the one that we have included. Next, $\kappa$ is a (possibly dimensionful) constant which allows us to easily compare our results with standard ones for SSGR and 2dDG. Finally, the potential term is

$$
V=\alpha X^{+} X^{-}+\lambda^{2} V_{0}
$$

where $\lambda$ is a constant with units of inverse length and $\alpha=\alpha(X), V_{0}=V_{0}(X)$. The "targetspace coordinates" [5, 6] $\left(X^{a}, X\right)$ have transparent geometric meanings. The special case of SSGR suggests that we may interpret $X$ as essentially the squared areal radius. Moreover, for SSGR the $X^{a}$ turn out to be (apart from $X$-dependent factors) the expansions associated with the null zweibein. In addition to this special-case interpretation, the fields $X^{a}$ are also closely related to the spacetime torsion when torsion is present $\left(\partial V / \partial X^{a} \neq 0\right)$. Indeed, among the Euler-Lagrange equations of motion (EOMs) associated with (1.1) is the following:

$$
\mathrm{d} e^{a}-\epsilon_{b}^{a} \omega \wedge e^{b}=\alpha X^{a} \epsilon .
$$

Therefore, one finds that $\alpha X^{a}={ }^{*} T^{a}:=\frac{1}{2} \epsilon^{\mu \nu} e^{a}{ }_{\lambda} T^{\lambda}{ }_{\mu \nu}$ is the Hodge dual of the torsion 2-form.

\section{Conformal transformation between physical and unphysical spacetimes}

In the absence of matter the EOMs associated with the ECM action (1.1) can be solved exactly for integrable $\alpha(X)$ and $v(X)$ [5 7]. In this paper, (1.1) (possibly also with matter)

\footnotetext{
${ }^{3}$ We shall unapologetically switch back and forth between the index and abstract notation in order to use the best notation when it is fitting. As a rule, we reserve the abstract notation for differential forms and linear operations. Thus, for example, we write $e^{+}, \omega[n]$, and $n[X]$, respectively, in place of $e^{+}{ }_{\mu} \mathrm{d} x^{\mu}, \omega_{\mu} n^{\mu}$, and $n^{\mu} \partial_{\mu} X$ (obviously in these examples $n$ is a vector field and $X$ is a scalar function). However, in order to avoid confusion, we use the index notation for vector fields, like $e_{+}{ }^{\mu}$ and $n^{\mu}$; and, therefore, (unless it appears in a linear operation as above) the plain letter $n$ is always the 1 -form $n_{\mu} \mathrm{d} x^{\mu}$.
} 
and its associated EOMs dictate the spacetime geometries we study, in particular determining (what we call) the unphysical metric $g_{\mu \nu}$. However, we have reason to be interested in certain physical metrics $\tilde{g}_{\mu \nu}$ which arise from the unphysical metrics $g_{\mu \nu}$ via conformal transformation. Our interest in the conformally related geometries stems from the local equivalence between a wide class of ECM models (even many with non-vanishing torsion [8]) and so-called generalized dilaton theories (GDTs) [16]. In the establishment of this equivalence, such GDTs emerge via the type conformal transformation we study here. Now, in fact, both SSGR and 2dDG are special cases of GDT. Therefore, the conformal transformations we consider now are essential, if we intend to apply our methods to (at least arguably) the most interesting $2 \mathrm{~d}$ models. For example, we shall write down a ECM action corresponding to vacuum SSGR. However, in this case it is the rescaled metric $\tilde{g}_{\mu \nu}$ rather than the metric $g_{\mu \nu}$ (determined directly by this particular ECM action's EOMs) which matches the familiar time-radial piece of the $4 \mathrm{~d}$ sBH line-element [5 7].

Our conformal transformations are given by the following set of field re-definitions in the action (using abstract notation):

$$
\begin{aligned}
e^{a} & =\exp (\varphi) \tilde{e}^{a} \\
X^{a} & =\exp (-\varphi) \tilde{X}^{a} \\
\omega & =\tilde{\omega}-(\mathrm{d} \varphi / \mathrm{d} X)^{\star} \mathrm{d} X,
\end{aligned}
$$

where $\varphi=\varphi(X)$ is at this point an arbitrary function of $X$ and the " $\star$ " represents the Hodgeduality associated with physical metric $\tilde{g}_{\mu \nu}$. The transformation (1.5) has been tailored to ensure that

$$
T_{\sigma \mu}^{\lambda}=\tilde{T}_{\sigma \mu}^{\lambda}
$$

is a consistent behavior for the torsion tensor under the transformation. Under the conformal transformation (1.5), the ECT action (1.1) becomes

$$
\tilde{L}=\kappa \int_{\mathcal{M}}\left[-\tilde{X}_{a} \tilde{D} \tilde{e}^{a}+X \mathrm{~d} \tilde{\omega}+(\mathrm{d} \varphi / \mathrm{d} X) \mathrm{d} X \wedge{ }^{\star} \mathrm{d} X-\tilde{V} \tilde{\epsilon}\right]-\kappa \oint_{\partial \mathcal{M}} X \tilde{\omega}+L^{(m)},
$$

with $\tilde{V}$ determined by the previous transformation rule $(1.5 \mathrm{~b})$ as well as $V_{0}=\exp (-2 \varphi) \tilde{V}_{0}$. The boundary term that we have included in (1.1) ensures that the variational principle associated with the rescaled action (1.7) features fixation of the same boundary data as fixed in the original variational principle. In the variational principle associated with (1.1) $e^{a}{ }_{\mu}$ and $X$ (and matter) are fixed, while in the variational principle associated with (1.7) $\tilde{e}^{a}{ }_{\mu}$ (which is built from $e^{a}{ }_{\mu}$ and $X$ ) and $X$ (and matter) are fixed. Preservation of the boundary conditions under the conformal rescaling is the main motivation for including this boundary term, although this requirement alone does not uniquely determine the choice of boundary term. Indeed, in $\S \mathrm{V}$ we shall add further boundary terms to the action which are insensitive to the conformal transformation.

\footnotetext{
${ }^{4}$ Chan et al have carefully considered conformal transformations and the role played by quite similar boundary terms in general $n$-dimensional dilaton theories. [19] In $\S \mathrm{V}$ we comment further on the relationship between the terms we consider and those found in that reference.
} 
The form (1.7) of the conformally rescaled action affords a rather direct comparison with the second-order action associated with a GDT. For simplicity here only, we restrict ourselves to vanishing torsion. Indeed, with $\partial V / \partial X^{a}=0$ (no torsion), elimination of the fields $\tilde{X}^{a}$ via their algebraic EOMs casts (1.7) into the following form:

$$
\begin{aligned}
\tilde{L}= & \frac{1}{2} \kappa \int_{\mathcal{M}} \mathrm{d}^{2} x \sqrt{-\tilde{g}}\left[X \tilde{\mathcal{R}}-2(\mathrm{~d} \varphi / \mathrm{d} X) \tilde{g}^{\mu \nu} \partial_{\mu} X \partial_{\nu} X-2 \lambda^{2} \exp (2 \varphi) V_{0}\right] \\
& +\kappa \oint_{\partial \mathcal{M}} \mathrm{d} x \sqrt{\left|\tilde{g}^{1}\right|} X \tilde{\epsilon}^{\mu \nu} \tilde{\omega}_{\mu} \tilde{\mathrm{n}}_{\nu}+L^{(m)},
\end{aligned}
$$

where we have switched to index notation, $\tilde{\mathcal{R}}$ is the Ricci scalar of $\tilde{g}_{\mu \nu}$, and $\tilde{g}^{1}$ is the determinant of the induced metric on $\partial \mathcal{M}$ (the absolute value sign is needed to handle timelike elements of $\partial \mathcal{M}$ ). Further, $\tilde{\mathrm{n}}_{\mu}$ is the 1 -form dual (in the metric $\tilde{g}_{\mu \nu}$ ) to the outwardpointing unit-normal vector field of the boundary $\partial \mathcal{M}$ as embedded in $\mathcal{M}\left(\tilde{\mathrm{n}}^{\mu}\right.$ differs from the unit normal by a sign on a spacelike element of $\partial \mathcal{M})$. We note that, in general, the boundary term in (1.8) can not be expressed purely in terms of the metric $\tilde{g}_{\mu \nu}$ (rather, it is at heart a zweibein expression). Therefore, as GDTs are, strictly speaking, metric theories, the action above is not quite appropriate to describe a GDT. We shall improve upon this situation in $\S \mathrm{V}$, where we begin with the addition of a boundary term to the ECM action which is zweibein-gauge invariant (in a sense to be made precise below).

Let us quickly consider the special case of vacuum SSGR, as it will determine some of our conventions. Set $L^{(m)}=0$ and make the following substitutions in (1.8):

$$
\begin{aligned}
\kappa^{S S G R} & =\lambda^{-2} \\
V_{0}^{S S G R} & =-\sqrt{\frac{1}{8}} X^{-1 / 2} \\
2 \varphi^{S S G R} & =\log \sqrt{2 X} .
\end{aligned}
$$

Finally, set $X=\frac{1}{2} \lambda^{2} R^{2}$, where $R(r, t)$ is the round 2-sphere areal radius (or luminosity parameter). Notice that $X$ is restricted to positive values. The minus sign in $(1.9 \mathrm{~b})$ cancels one in (1.8). We have introduced this spurious minus sign only to ensure that our conventions are more in harmony with those of Refs. [6] (although those references do adopt a different metric-signature convention). The result of these substitutions is an action which (as was first noted by Thomi, Isaak, and Hájíček [2]) has as its associated EOMs the 4d Einstein equations subject to the ansatz of spherical symmetry. In addition, the resulting action can (apart from certain technical points concerning the zweibein dependence of the boundary term) be obtained directly form the $4 \mathrm{~d}$ Einstein-Hilbert action via a reduction by spherical symmetry (see, for example, [20]). The choices (1.9) ensures that the ADM mass as measured at spatial infinity corresponds to the SBH mass parameter. Moreover, the choice (1.9a) gives the action the units of action in $4 \mathrm{~d}$.

SSGR is an example of the "physical" situation that we are interested in. Namely, we shall require that a flat line-element ("Minkowski groundstate") is obtained when the mass parameter of the solution is set to zero. We consider the most general class of such models in $\S$ IV. It should be emphasized that the conformal transformations used in this paper leave minimal interactions with a scalar field invariant. A minimally coupled fermion transforms covariantly. 


\section{The first-order approach}

We stress that we do not examine in detail the EOMs associated with the action (1.8), or, for that matter, those associated with the action (1.7). Indeed, since these EOMs are considerably more complicated than those associated with (1.1), it is prudent to work directly at the level of the ECM action (11.1) with its relatively simple first-order EOMs; switching via conformal transformation to the hatted variables of real interest only "at the end of the day." The merit of this approach is strikingly evident in the transparent derivation of the conservation law (always present for the $2 \mathrm{~d}$ theories we study here) [7]. Indeed, a simple linear combination of the EOMs obtained by varying the ECM action (1.1) with respect to the zweibein and spin connection yields the promised conservation law, namely,

$$
\mathrm{d}\left[X^{+} X^{-} \exp (\alpha X)+W\right]+U^{(m)}=0,
$$

where for simplicity here we assume $\alpha$ is constant and

$$
W=\lambda^{2} \int_{X_{0}}^{X} V_{0}(y) \exp (\alpha y) \mathrm{d} y .
$$

In the above conservation law $U^{(m)}$ arises from the presence of matter. Now, to make (1.10) look like a conservation law, we appeal to the Poincaré-Lemma which tells us that that $U^{(m)}=\mathrm{d} C^{(m)}$ (at least locally), with $C^{(m)}$ a field-nonlocal expression built from the (geometric variables and) matter fields. That $U^{(m)}$ is locally exact must also follow via a clever combination of the remaining (notably matter) EOMs. Therefore, we have found

$$
\mathrm{d}\left[C+C^{(m)}\right]=\mathrm{d}\left[X^{+} X^{-} \exp (\alpha X)+W+C^{(m)}\right]=0
$$

for the conservation law valid for all ECMs in 2d. [5 -7] It is, of course, trivial to rewrite $C$ in terms of the physical variables. For the case of vacuum SSGR we shall see that $M_{S}=$ $-\lambda^{-3} C^{S S G R}$ (mass dimension 1 in $4 \mathrm{~d}$ ) corresponds to the mass parameter of the SBH. (Note that $C$ has mass dimension -2 in $4 \mathrm{~d}$, and, hence, the need for the $\lambda^{-3}$ factor is evident.)

\section{ADM DECOMPOSITIONS AND KINEMATICS FOR ZWEIBEINE}

Let us now provide a more detailed description of the boundary structure $\partial \mathcal{M}$ associated with our spatially bounded spacetime region $\mathcal{M}$. The region $\mathcal{M}$ consists of a collection of one dimensional spacelike slices $\Sigma$. The letter $\Sigma$ denotes both the foliation of $\mathcal{M}$ into spacelike slices and a generic leaf of the foliation. The initial spacelike slice is $\Sigma^{\prime}$ (the curve of constant coordinate time $t=t^{\prime}$ ), and, likewise, the final spacelike slice is $\Sigma^{\prime \prime}$ (the curve of constant coordinate time $\left.t=t^{\prime \prime}\right)$. On spacetime $\mathcal{M}$ we have coordinates $(t, r)$, and a generic spacetime point is $B(t, r)$. Every constant- $t$ slice $\Sigma$ has two boundary points $B_{i}$ (at $r=r_{i}$ ) and $B_{o}$ (at $r=r_{o}$ ). Assume that along $\Sigma$ the coordinate $r$ increases monotonically from $B_{i}$ to $B_{o}$. We represent the timelike history $B_{i}(t) \equiv B\left(t, r_{i}\right)$ by $\overline{\mathcal{T}}_{i}$ and refer to it as the inner boundary. Likewise, we represent the timelike history $B_{o}(t) \equiv B\left(t, r_{o}\right)$ by $\overline{\mathcal{T}}_{o}$ and refer to it as the 
outer boundary. We denote the corner points of our spacetime as follows: $B_{i}^{\prime} \equiv B\left(t^{\prime}, r_{i}\right)$, $B_{o}^{\prime} \equiv B\left(t^{\prime}, r_{o}\right), B_{i}^{\prime \prime} \equiv B\left(t^{\prime \prime}, r_{i}\right)$, and $B_{o}^{\prime \prime} \equiv B\left(t^{\prime \prime}, r_{o}\right)$.

We can also consider a radial foliation of $\mathcal{M}$ by a family of 1-dimensional timelike slices which extend from $\overline{\mathcal{T}}_{i}$ outward to $\overline{\mathcal{T}}_{o}$ (here the $\mathcal{T}$ 's stand for "time" and they are "barred" for reasons which become clear) [23]. These are constant- $r$ curves in $\mathcal{M}$. Like before, we loosely use the letter $\overline{\mathcal{T}}$ both to denote the radial foliation and a generic leaf of this foliation. Abusing the notation a bit, we also often let the symbol $\overline{\mathcal{T}}$ denote the total timelike boundary $\overline{\mathcal{T}}_{i} \cup \overline{\mathcal{T}}_{o}$. However, when the symbol $\overline{\mathcal{T}}$ has this meaning, it always appears in the phrase "the $\overline{\mathcal{T}}$ boundary." Fig. 1 depicts the geometry associated with the foliations of our spacetime patch $\mathcal{M}$.

\section{A. Metric decompositions}

The metric on a generic $\Sigma$ slice is $\Lambda^{2}$, and the metric on the $\overline{\mathcal{T}}$ boundary is denoted by $-\bar{N}^{2}$. In terms of the $\Sigma$ foliation, the metric may be written in ADM form [17]

$$
g_{\mu \nu} \mathrm{d} x^{\mu} \mathrm{d} x^{\nu}=-N^{2} \mathrm{~d} t^{2}+\Lambda^{2}\left(\mathrm{~d} r+N^{r} \mathrm{~d} t\right)^{2},
$$

with $N$ and $N^{r}$ denoting the familiar lapse and shift. The vector field

$$
u^{\mu} \partial / \partial x^{\mu}=N^{-1}\left(\partial / \partial t-N^{r} \partial / \partial r\right)
$$

is the unit, timelike, future-pointing normal to the $\Sigma$ foliation.

In terms of the $\overline{\mathcal{T}}$ foliation, the $\mathcal{M}$ metric takes the form

$$
g_{\mu \nu} \mathrm{d} x^{\mu} \mathrm{d} x^{\nu}=\bar{\Lambda}^{2} \mathrm{~d} r^{2}-\bar{N}^{2}\left(\mathrm{~d} t+\bar{\Lambda}^{t} \mathrm{~d} r\right)^{2},
$$

where $\bar{\Lambda}$ and $\bar{\Lambda}^{t}$ are the radial lapse and radial shift. The unit, spacelike, $\overline{\mathcal{T}}$-foliation normal is

$$
\bar{n}^{\mu} \partial / \partial x^{\mu}=\bar{\Lambda}^{-1}\left(\partial / \partial r-\bar{\Lambda}^{t} \partial / \partial t\right) .
$$

On the outer boundary $\overline{\mathcal{T}}_{o}$ the outward normal is $\bar{n}^{\mu}$, while on the inner boundary $\overline{\mathcal{T}}_{i}$ the outward normal is $-\bar{n}^{\mu}$.

By equating the coefficients of the above forms of $g_{\mu \nu}$, we obtain the following relations between the "barred" and "unbarred" variables:

$$
\begin{aligned}
\bar{N} & =N / \gamma \\
\bar{\Lambda} & =\gamma \Lambda \\
\Lambda N^{r} / N & =-\bar{N} \bar{\Lambda}^{t} / \bar{\Lambda} .
\end{aligned}
$$

\footnotetext{
${ }^{5}$ Were we to adopt "thermodynamic boundary conditions" [21,22, 20] for the black-hole solutions we consider, we would "seal" the inner boundary. In other words, the time development at the inner boundary would be arrested, and the point $B_{i}$ would correspond to a bifurcation point in a Kruskal-like diagram. Although we do not consider such boundary conditions in this work, we make mention of them, since we do go some of the way towards setting them up.
} 
Here $\gamma:=\left(1-v^{2}\right)^{-1 / 2}$ is the local relativistic factor associated with the velocity $v:=$ $\Lambda N^{r} / N=-\bar{N} \bar{\Lambda}^{t} / \bar{\Lambda}=:-\bar{v}$ 23,20,24]. The timelike normal associated with the foliations $B_{i}(t)$ and $B_{o}(t)$ of the boundary slices $\overline{\mathcal{T}}_{i}$ and $\overline{\mathcal{T}}_{o}$ is $\bar{u}^{\mu} \partial / \partial x^{\mu}=\bar{N}^{-1} \partial / \partial t$. Note that on the $\overline{\mathcal{T}}$ boundary the vector fields $u^{\mu}$ and $\bar{u}^{\mu}$ need not coincide. Also, fixation of the $t$ coordinate gives a collection of points $B(r)$ which foliates the slice $\Sigma$. The normal associated with this foliation of $\Sigma$ is $n^{\mu} \partial / \partial x^{\mu}=\Lambda^{-1} \partial / \partial r$. At the inner boundary $-n^{\mu}$ is the outward-pointing normal of $B_{i}$ as embedded in $\Sigma$, while at the outer boundary $n^{\mu}$ is the outward-pointing normal of $B_{o}$ as embedded in $\Sigma$. On the inner and outer boundaries $n^{\mu}$ and $\bar{n}^{\mu}$ need not coincide. It is easy to verify that we have

$$
\begin{aligned}
& \bar{u}^{\mu}=\gamma u^{\mu}+v \gamma n^{\mu} \\
& \bar{n}^{\mu}=\gamma n^{\mu}+v \gamma u^{\mu}
\end{aligned}
$$

as the point-wise boost relations between the "barred" and "unbarred" frames.

With the 1 -forms $u=u_{\mu} \mathrm{d} x^{\mu}$ and $n=n_{\mu} \mathrm{d} x^{\mu}$ we write down a general null spacetime zweibein, namely,

$$
\begin{aligned}
& e^{+}=\sigma_{L} e^{\rho} \sqrt{\frac{1}{2}}(-u+n)=\sigma_{L} e^{\rho} \sqrt{\frac{1}{2}}\left[\left(N+\Lambda N^{r}\right) \mathrm{d} t+\Lambda \mathrm{d} r\right] \\
& e^{-}=\sigma_{L} e^{-\rho} \sqrt{\frac{1}{2}}(-u-n)=\sigma_{L} e^{-\rho} \sqrt{\frac{1}{2}}\left[\left(N-\Lambda N^{r}\right) \mathrm{d} t-\Lambda \mathrm{d} r\right]
\end{aligned}
$$

where $\sigma_{L}= \pm 1$ (" $L$ " for "Lorentz") and $\rho$ (an arbitrary point-wise boost factor) ensure that the zweibein is completely general. In terms of the 1 -forms $\bar{u}=\bar{u}_{\mu} \mathrm{d} x^{\mu}$ and $\bar{n}=\bar{n}_{\mu} \mathrm{d} x^{\mu}$ our general zweibein is given by

$$
\begin{aligned}
& e^{+}=\sigma_{L} e^{\bar{\rho}} \sqrt{\frac{1}{2}}(-\bar{u}+\bar{n})=\sigma_{L} e^{\bar{\rho}} \sqrt{\frac{1}{2}}\left[\bar{N} \mathrm{~d} t+\left(\bar{N} \bar{\Lambda}^{t}+\bar{\Lambda}\right) \mathrm{d} r\right] \\
& e^{-}=\sigma_{L} e^{-\bar{\rho}} \sqrt{\frac{1}{2}}(-\bar{u}-\bar{n})=\sigma_{L} e^{-\bar{\rho}} \sqrt{\frac{1}{2}}\left[\bar{N} \mathrm{~d} t+\left(\bar{N} \bar{\Lambda}^{t}-\bar{\Lambda}\right) \mathrm{d} r\right]
\end{aligned}
$$

with $\bar{\rho}=\rho+\eta$. The parameter $\eta=\frac{1}{2} \log |(1+v) /(1-v)|$ is associated with the local boost between $u$ and $\bar{u}$. By construction, $\rho, \bar{\rho}$, and $\eta$ are everywhere finite and well-defined.

\section{B. Killing vector}

For the models we study with the ECM action, (at least in vacuo) the corresponding solution $g_{\mu \nu}$ will possess a Killing field $k^{\mu}$; and we shall be quite interested in the preferred foliation of spacetime which is associated with $k^{\mu}$. We use $\hat{\Sigma}$ both to denote this foliation and to represent a generic leaf thereof. Note that this foliation may have certain pathologies within the finite patch $\mathcal{M}$ under examination. As experience with the Schwarzschild geometry suggests, $k^{\mu}$ need not be a timelike vector everywhere on our spacetime region. In general, a maximal extension of the solution $g_{\mu \nu}$ will include static and dynamical regions separated by horizons. A priori we want to allow for all possibilities: that our spacetime patch $\mathcal{M}$ lies entirely within a dynamical region, that it lies entirely within a static region, and that it covers portions of both static and dynamical regions. Later on in $\S$ VI we shall find it necessary to assume that both of the timelike boundary elements, $\overline{\mathcal{T}}_{i}$ and $\overline{\mathcal{T}}_{o}$, lie entirely within static regions. 
For vacuum, an equation of motion [namely, (3.1]c) given later] implies that $X^{ \pm}=$ $\pm e_{\mp}[X]$. Now, in fact, the vector fields ${\sigma_{L}}_{e_{ \pm}}{ }^{\mu}$ are by construction everywhere future-pointing in our formalism. Hence, with the Penrose diagram for the SBH and the above formulae as guides, we set $X^{ \pm}=-\sigma_{ \pm} \sigma_{L}\left|X^{ \pm}\right|$(defining $\sigma_{ \pm}$) and introduce spacetime regions of type I, II, III, and IV as follows:

$$
\begin{aligned}
\text { I, } & \sigma_{+}=1=\sigma_{-} \\
\text {II, } & \sigma_{+}=1=-\sigma_{-} \\
\text {III, } & \sigma_{+}=-1=\sigma_{-} \\
\text {IV }, & \sigma_{+}=-1=-\sigma_{-} .
\end{aligned}
$$

Here the SBH has been used only to elucidate the appropriate choices. Analogous ones are made for the (possibly quite complex) Penrose diagram corresponding to a given general ECM. Let $\hat{u}^{\mu}$ represent the normalized Killing direction, i. e.

$$
\hat{u}^{\mu}=\left|k_{\lambda} k^{\lambda}\right|^{-1 / 2} k^{\mu}
$$

and pick its orthogonal partner $\hat{n}^{\mu}$ such that in a static region the pair $\left(\sigma_{+} \hat{u}^{\mu}, \sigma_{-} \hat{n}^{\mu}\right)$ has the same orientation as $\left(u^{\mu}, n^{\mu}\right)$. A static region is determined by $\sigma_{K}:=-\operatorname{sign}\left(k_{\mu} k^{\mu}\right)=1$ ("K" for "Killing"). Notice that $\sigma_{+} \sigma_{-}=\sigma_{K}$. For a static region $\sigma_{+} \hat{u}^{\mu}$ is the unit futurepointing 2-velocity of Eulerian observers which ride along the orbits of the isometry and are instantaneously at rest in the $\hat{\Sigma}$ slices. However, in a dynamical region $\left(\sigma_{K}=-1\right)$ we must exchange the roles of $\hat{u}^{\mu}$ and $\hat{n}^{\mu}$. In a dynamical region it is the pair $\left(\sigma_{-} \hat{n}^{\mu}, \sigma_{+} \hat{u}^{\mu}\right)$ which has the same orientation as $\left(u^{\mu}, n^{\mu}\right)$. Therefore, we find

$$
\begin{aligned}
& \hat{u}^{\mu}=\frac{1}{2}\left(\sigma_{+}+\sigma_{-}\right)\left(\hat{\gamma} u^{\mu}+\hat{v} \hat{\gamma} n^{\mu}\right)+\frac{1}{2}\left(\sigma_{+}-\sigma_{-}\right)\left(\hat{\gamma} n^{\mu}+\hat{v} \hat{\gamma} u^{\mu}\right) \\
& \hat{n}^{\mu}=\frac{1}{2}\left(\sigma_{-}+\sigma_{+}\right)\left(\hat{\gamma} n^{\mu}+\hat{v} \hat{\gamma} u^{\mu}\right)+\frac{1}{2}\left(\sigma_{-}-\sigma_{+}\right)\left(\hat{\gamma} u^{\mu}+\hat{v} \hat{\gamma} n^{\mu}\right)
\end{aligned}
$$

as the boost relations between the $\Sigma$ Eulerian observers and the $\hat{\Sigma}$ Eulerian observers. Here, like before, $\hat{v}$ is a local boost velocity and $\hat{\gamma}=\left(1-\hat{v}^{2}\right)^{-1 / 2}$ is the associated relativistic factor. Note that our construction breaks down on a horizon, characterized by $k_{\mu} k^{\mu}=0$. Therefore, we may write the arbitrary null zweibein as

$$
\begin{aligned}
& e^{+}=\sigma_{L} e^{(\rho+\hat{\eta})} \sqrt{\frac{1}{2}}\left(-\sigma_{-} \hat{u}+\sigma_{+} \hat{n}\right) \\
& e^{-}=\sigma_{L} e^{-(\rho+\hat{\eta})} \sqrt{\frac{1}{2}}\left(-\sigma_{+} \hat{u}-\sigma_{-} \hat{n}\right),
\end{aligned}
$$

where the parameter $\hat{\eta}=\frac{1}{2} \log |(1+\hat{v}) /(1-\hat{v})|$, very important for our purposes, describes the local boost between the $\Sigma$ and $\hat{\Sigma}$ observers. We note the $\hat{\eta}$ is everywhere well-behaved and finite except on a horizon where it is $\pm \infty$. For a Kruskal-like diagram, the value of $\hat{\eta}$ at the bifurcation point has a direction-dependent limit. Notice that the sign factors $\sigma_{ \pm}$ present in (2.11) ensures that the expression

$$
g:=-e^{+} \otimes e^{-}-e^{-} \otimes e^{+}=\sigma_{K}(-\hat{u} \otimes \hat{u}+\hat{n} \otimes \hat{n})
$$

for the spacetime metric has the correct signature in all regions. So far we have a threefold non-degeneracy in our formalism. We have considered three distinct spacetime frames, corresponding to $\Sigma, \bar{\Sigma}$, and $\hat{\Sigma}$. Of course, this non-degeneracy carries over to the physical or "tilde" geometry as well. 


\section{EQUATIONS OF MOTION AND CONSERVATION LAW}

Taking the simple case when $\alpha$ is a constant (which, as we show later, results in no loss of generality for our purposes), we find the following EOMs corresponding to the ECM action (1.1): [7]

$$
\begin{aligned}
\mathrm{d} X^{ \pm} \pm \omega X^{ \pm} & = \pm e^{ \pm} V+A^{(m) \pm} \\
\mathrm{d} e^{ \pm} \pm \omega \wedge e^{ \pm} & =-\alpha X^{ \pm} e^{+} \wedge e^{-} \\
\mathrm{d} X & =X^{+} e^{-}-X^{-} e^{+}+A^{(m)} \\
\mathrm{d} \omega & =-\lambda^{2}\left(\mathrm{~d} V_{0} / \mathrm{d} X\right) e^{+} \wedge e^{-}+B^{(m)},
\end{aligned}
$$

where one also has matter EOMs obtained by varying the matter fields in $L^{(m)}$. The terms $A^{(m) \pm}, A^{(m)}$, and $B^{(m)}$ are produced by varying the matter action $L^{(m)}$ with respect to $e^{ \pm}, \omega$, and $X$, respectively. Notice that $B^{(m)}$ is non-zero whenever the matter contribution to the action depends on $X$, as is the case for ssGR. Minimally coupled scalar fields and fermions in $2 \mathrm{~d}$ have $A^{(m)}=0$. Moreover, for such a scalar field $S$, the the matter contributions in (3.1a) are $A^{(m) \pm}=\mp\left[^{*}\left(e^{ \pm} \wedge \mathrm{d} S\right)\right] e^{\mp}$ (note that the quantity enclosed by square brackets is a scalar). For our purposes it suffices only to note that the terms $A^{(m) \pm}, A^{(m)}$, and $B^{(m)}$ may be present, thereby providing contributions to $C^{(m)}$ in (1.12). As described in the introduction, starting with (3.1a) and (3.1c), one obtains (1.10), from which immediately follows the "absolute" conservation law (1.12). This derivation shows that it is the quantity

$$
C=X^{+} X^{-} \exp (\alpha X)+W
$$

and only this quantity, which is affected by the influx of matter [7]. Regarding the presence of matter, for the following we only need this piece of information from the full EOMs.

The most general vacuum solution [5 7.25] to the EOMs (3.1) is readily obtained

$$
\begin{aligned}
e^{-} & =-\exp (\alpha X) X^{-} \mathrm{d} f \\
e^{+} & =-\mathrm{d} X / X^{-}-X^{+} \exp (\alpha X) \mathrm{d} f \\
\omega & =\mathrm{d} X^{-} / X^{-}-\exp (\alpha X) V \mathrm{~d} f
\end{aligned}
$$

in terms of arbitrary functions $f, X, X^{ \pm}$(see Ref. [7] for a simple derivation). Here $X^{+}$ must be expressed in terms of $C$ by using (3.2). The form (3.3) of the solution is valid for $X^{-} \neq 0$. A similar form with $X^{-} \leftrightarrow X^{+}$allows for the description of $X^{+} \neq 0$ patches (possibly including points where $X^{-}=0$ ). The resulting line-element

$$
(\mathrm{d} s)^{2}=-2 \exp (\alpha X)\left[(C-W) \mathrm{d} f^{2}+\mathrm{d} f \mathrm{~d} X\right]
$$

is expressed in what are essentially Eddington-Finkelstein coordinates. The quantity

$$
k^{\lambda} k_{\lambda}=-2 \exp (\alpha X)(C-W)
$$

coincides with the norm of the Killing vector field $k^{\mu}\left[=(\partial / \partial f)^{\mu}\right.$ in the chosen coordinates], and the presence and type of horizon associated with the line-element (3.4) can be simply read off from of the zeros in (3.5). Notice that $C-W=\sigma_{K}|C-W|$ (cf. $\S$ II.B). 
Consider again the Schwarzschild example (vacuum SSGR), where we have set $C^{S S G R}=$ $-\lambda^{3} M_{S}$ and $X=\frac{1}{2} \lambda^{2} R^{2}$. From (1.9b) and the definition of $W$, we find $W^{S S G R}=-\frac{1}{2} \lambda^{3} R$. Therefore, in the vacuum SSGR case (3.4) takes the form

$$
(\mathrm{d} s)^{2}=-\lambda R\left[\left(1-2 M_{S} / R\right) \mathrm{d} u^{2}+2 \mathrm{~d} u \mathrm{~d} R\right]
$$

in terms of the retarded-time coordinate $u=\lambda f$. Upon our conformal rescaling $(\mathrm{d} s)^{2}=$ $\exp (2 \varphi)(\mathrm{d} \tilde{s})^{2}$ with $\exp \left(2 \varphi^{S S G R}\right)=\lambda R$ from $(1.9 \mathrm{c})$, we obtain $(\mathrm{d} \tilde{s})^{2}$, the time-radial piece of the SBH line-element in outgoing EF coordinates [26]. We make the important observation that for SSGR the conformal factor is $\exp \left(2 \varphi^{S S G R}\right)=2 \lambda^{-2}\left|W^{S S G R}\right|$. One recovers the DBH line-element in a similar fashion. [6. []

By comparing our expressions for $e^{ \pm}$in terms of the ADM variables with the general solution (3.3), we obtain the following expressions for the $\Sigma$ metric, lapse, and shift

$$
\begin{aligned}
\Lambda^{2} & =-2 \exp (\alpha X) f^{\prime}\left[X^{\prime}+(C-W) f^{\prime}\right] \\
N & =\exp (\alpha X) \Lambda^{-1}\left[\dot{f} X^{\prime}-\dot{X} f^{\prime}\right] \\
N^{r} & =-\exp (\alpha X) \Lambda^{-2}\left[\dot{X} f^{\prime}+\dot{f} X^{\prime}+2(C-W) \dot{f} f^{\prime}\right] .
\end{aligned}
$$

Let us now verify that $N$ and $\Lambda^{2}$ are everywhere positive, which should follow by our assumption that the everywhere-spacelike slices of the $\Sigma$ foliation everywhere advance into the future. To this end, let $f=\tau-X^{*}$, where $X^{*}$ is a tortoise-type coordinate [26] obeying $\partial X^{*} / \partial X=\frac{1}{2}(C-W)^{-1}$; and consider the coordinates $(\tau, X)$. As experience with the Schwarzschild geometry suggests, in a static region $\left(\sigma_{K}=1\right)$ we know that either the coordinate set $(\tau, X)$ or $(-\tau,-X)$ will have the same orientation as the set $(t, r)$ of coordinates associated with the $\Sigma$ foliation. In a dynamical region either the set $(-X, \tau)$ or $(X,-\tau)$ will have the same orientation as $(t, r)$. Using these coordinates along with the sign of the Killing norm (3.5), one may straightforwardly verify that both $N$ and $\Lambda^{2}$ are everywhere positive in all regions covered by our spacetime patch $\mathcal{M}$.

\section{DILATON MODELS WITH MINKOWSKI GROUND STATE}

Klösch and Strobl have carried out a comprehensive global analysis of all vacuum $2 \mathrm{~d}$ gravity models based upon the general solution (3.3) [6]. Their results, as well as previous ones obtained from the global analysis of " $R^{2}+T^{2}$ gravity" [27], clearly demonstrate that, in general, the singularity structures for such models exhibit little similarity with the $2 \mathrm{~d}$ singularity structures familiar from SSGR, such as the SBH and RNBH. In particular, a flat (or at least de Sitter) "ground state" for certain values of $C$ is not realized. Moreover, even asymptotically flat (or asymptotically de Sitter) solutions are difficult to realize. However, as mentioned, by a (local) re-definition of fields it has been shown that ECM models [even those with torsion, $\alpha \neq 0$ in (1.3)] are equivalent to torsion-free GDTs. [8] In this correspondence between ECMs and GDTs, the resulting change in global properties gives rise to the appearance of asymptotically flat solutions.

For some time, the DBH from 2dDG [9-11] has attracted special interest because several of its features, namely, asymptotic flatness and a Minkowski ground state for $C=0$, mimic 
those associated with the SBH. Moreover, the DBH in the presence of matter is also an exactly solvable model. Recently, however, it has been shown that the original DBH resembles the real SBH rather superficially, in that it possesses complete null geodesics [12]. In Ref. [12], a family of models has been considered which are characterized by one bifurcate Killing horizon as well as incomplete non-null and null geodesics. In other words, each model in the family closely resembles the SBH (which is, in fact, a member of the class itself) and possesses a "genuine" SBH Penrose diagram. We would like to point out that these models are among the GDTs we study here.

\section{A. Physical dilaton models}

Motivated by the fact that theories with torsion are (locally) equivalent to (globally better-behaved) generalized dilaton models, we consider only the torsion-less case here $[\alpha=0$ in (1.3)]. As we shall explicitly demonstrate later in $\S$ VI.A, this assumption results in no loss of generality at the level of GDTs (i. e. after conformal transformation). If we demand that the line-element for $C=0$ reduces to the one for flat spacetime, then we find an (essentially) unique conformal transformation of (3.3). Namely, the one determined by

$$
2 \varphi=\log \left(2 \lambda^{-2}|W|\right)
$$

(the factor of 2 under the square root is chosen to have agreement with the standard conventions for SSGR) which leads to the following rescaled metric:

$$
(\mathrm{d} \tilde{s})^{2}=-\left[\left(\sigma_{W}+C|W|^{-1}\right) \lambda^{2} \mathrm{~d} f^{2}+2 \mathrm{~d} f \mathrm{~d} \tilde{X}\right] .
$$

Here we have set $W=-\sigma_{W}|W|$ and defined $\tilde{X}$ by

$$
\mathrm{d} \tilde{X} / \mathrm{d} X=\frac{1}{2} \lambda^{2}|W|^{-1} .
$$

One obtains an asymptotically flat line-element for models with $\sigma_{W}=1$ (and, hence, negative $W)$ and $\lim _{X \rightarrow \infty}|W|=\infty$. Henceforth, we shall be most interested in the most general class of such models. The models in this general class are the ones that we refer to as "physical" (cf. the discussion in $\S$ I.A and I.C). In terms of the new coordinate $\tilde{X}$, we rewrite the GDT action (1.8) in true "dilaton form,"

$$
\begin{aligned}
\left.\tilde{L}\right|^{\text {dil }}= & \frac{1}{2} \kappa \int_{\mathcal{M}} \mathrm{d}^{2} x \sqrt{-\tilde{g}}\left[X \tilde{\mathcal{R}}-\left(\mathrm{d}^{2} X / \mathrm{d} \tilde{X}^{2}\right)\left(\tilde{g}^{\mu \nu} \partial_{\mu} \tilde{X} \partial_{\nu} \tilde{X}-\lambda^{2}\right)\right] \\
& +\kappa \oint_{\partial \mathcal{M}} \mathrm{d} x \sqrt{\left|\tilde{g}^{1}\right|} X \tilde{\epsilon}^{\mu \nu} \tilde{\omega}_{\mu} \tilde{\mathrm{n}}_{\nu}+L^{(m)} .
\end{aligned}
$$

In this action one should consider $X=X(\tilde{X})$. As mentioned, unless we employ a suitable gauge choice for the zweibein at the boundary $\partial \mathcal{M}$, the action (4.4) cannot be viewed solely as a metric action. With strict attention paid to this point, we examine the canonical form of this action in $\S$ VI.A.

Using our earlier results (3.7) we may obtain expressions for the rescaled $\Sigma$ metric $\tilde{\Lambda}^{2}=$ $\frac{1}{2} \lambda^{2}|W|^{-1} \Lambda^{2}$, lapse $\tilde{N}=\sqrt{\frac{1}{2}} \lambda|W|^{-1 / 2} N$, and shift $\tilde{N}^{r}=N^{r}$. Indeed, a short calculation yields the following set: 


$$
\begin{aligned}
\tilde{\Lambda}^{2} & =-f^{\prime}\left[2 \tilde{X}^{\prime}+\left(1+C|W|^{-1}\right) \lambda^{2} f^{\prime}\right] \\
\tilde{N} & =\tilde{\Lambda}^{-1}\left[\dot{f} \tilde{X}^{\prime}-\dot{\tilde{X}} f^{\prime}\right] \\
N^{r} & =-\tilde{\Lambda}^{-2}\left[\dot{\tilde{X}} f^{\prime}+\dot{f} \tilde{X}^{\prime}+\left(1+C|W|^{-1}\right) \lambda^{2} \dot{f} f^{\prime}\right] .
\end{aligned}
$$

Now, since conformal transformations preserve casual structure, we need not check the signs of $\tilde{N}$ and $\tilde{\Lambda}^{2}$ as we did with the unphysical lapse and $\Sigma$ metric. We have written these equations in order to make the following derivation. Setting $C=0$ we obtain a flat lineelement. Therefore, we use coordinate freedom such that $(t, r)$ produce a manifestly flat line-element, $\left(\mathrm{d} \tilde{s}_{0}\right)^{2}=-\mathrm{d} t^{2}+\mathrm{d} r^{2}$. This implies that $\tilde{N}_{0}=\tilde{\Lambda}_{0}^{2}=1$ and $\tilde{N}_{0}^{r}=0$. Enforcing these conditions on the set (4.5), we obtain

$$
\tilde{X}_{0}=\lambda r .
$$

Therefore, the groundstates of the GDT models we consider are in fact "linear-dilaton vacua" as in the standard 2dDG. [10,11] The existence of such a groundstate plays a crucial role in our examination of asymptotic energy found in $\S$ VI.C. One also finds that $u_{0}:=\lambda f_{0}=t-\lambda^{-1} \tilde{X}_{0}$ as expected.

We should mention that the influx of particles into the groundstate with $C=0$ changes the state to $C \neq 0$. Therefore, as in all strictly $2 \mathrm{~d}$ models we encounter the well-known deficiency that there is no critical density below which an ingoing "radial" wave is simply reflected to an outgoing one (i. e. $C$ stays zero) so that no singularity is created. This would appear to be cured by the introduction of non-minimal couplings (e. g. involving an explicit $X$-dependence as in SSGR). In SSGR criticality has been observed in some analytic (albeit rather unphysical) solutions [28].

\section{B. Universal mass in GDT}

We conclude this section by discussing the concept of "universal mass" in GDT. With our conventions the well-known Misner-Sharp definition [14] of gravitational mass for SSGR reads as follows:

$$
M_{M S}=\frac{1}{2} R\left[1-\tilde{g}^{\mu \nu}\left(\partial_{\mu} R\right)\left(\partial_{\nu} R\right)\right],
$$

again where $R$ is the areal radius. This is the accepted definition of "quasilocal" gravitational mass in SSGR, and a wide array of quasilocal mass constructions from full $4 \mathrm{~d}$ general relativity yield this result in the special case of spherical symmetry. [15] Viewed as a canonical expression (cf. the discussion in $\S \mathrm{V}, \mathrm{VI}$ ), the Misner-Sharp mass plays a fundamental role in Kuchař's approach 四 to the canonical reduction of (vacuum) SSGR. 9 Such a "mass

\footnotetext{
${ }^{6}$ Kuchař's approach is the SBH version of the canonical reduction we shall study in $\S$ VI. A quite similar analysis was earlier carried out by Schaller and Strobl [29] in the context of the KatanaevVolovich model [27].
} 
canonical variable" had been earlier isolated and successfully used in the canonical reduction of SSGR by Kastrup and Thiemann. Their work is based mostly, but not entirely, on the Ashtekar gravitational variables [3]. Furthermore, Guven and Ó Murchadha's thorough investigation 30 of gravitational collapse in SSGR also makes use of this mass.

In our formalism a generalization of the Misner-Sharp definition, a universal mass function valid for all dilaton theories, appears quite naturally. Namely, the covariant expression for the quantity $C$ (absolutely conserved in vacuo). Again, set $\alpha=0$, which, to repeat, amounts to no loss of generality at the level of GDT. We seek a covariant version of this expression which is written in terms of the conformally rescaled geometry. A simple analysis of the based on the line-element (4.2) readily yields the desired expression,

$$
C=\frac{1}{4} \lambda^{2}|W|^{-1} \tilde{g}^{\mu \nu}\left(\partial_{\mu} X\right)\left(\partial_{\nu} X\right)-|W|
$$

With the SSGR choices (1.9) one may easily verify that $C^{S S G R}=-\lambda^{3} M_{M S}$, as expected. The covariant expression for $C$ takes an especially nice form in terms of the dilaton field $\tilde{X}$ introduced above, namely,

$$
C=|W|\left[\lambda^{-2} \tilde{g}^{\mu \nu}\left(\partial_{\mu} \tilde{X}\right)\left(\partial_{\nu} \tilde{X}\right)-1\right]
$$

For 2dDG this is Tada and Uehara's "local mass" 31, which has also been examined by Hayward and earlier by Frolov. [33,32 As a "mass canonical variable" the universal mass has also been consider by several authors in the context of GDT [34, 35].

\section{CANONICAL ACTION AND QUASILOCAL ENERGY-MOMENTUM}

In this section we begin our examination of the canonical formulation of both ECMs and GDTs for the bounded spacetime region $\mathcal{M}$. First, we present our variational principle, which is determined by adding certain further boundary terms to the ECM action, and then write the action in canonical form. In this section we also examine the notion of gravitational (quasilocal) energy and momentum for both ECMs and GDTs. Again, the vehicle for this examination is the first-order approach.

\section{A. Boundary terms and variational principle}

As mentioned, we are chiefly interested in those physical geometries $\left(\mathcal{M}, \tilde{g}_{\mu \nu}\right)$ introduced in $\S$ IV.A and obtained via the conformal transformation discussed in $\S$ I.C. We have argued that the addition of a boundary term to the base ECM action is necessary in order to preserve the variational principle under the conformal rescaling. I However, note that the naive boundary term (1.2) introduced in $\S$ I.C is in fact zweibein-gauge dependent, and

\footnotetext{
${ }^{7}$ We shall show in the next section, that from the canonical viewpoint the addition of a boundary term to the base ECM action is necessary in order to manifest the conformal rescaling as a canonical transformation.
} 
this breaks the local Lorentz invariance of the theory at the boundary. In this section we shall write down an "improved" boundary term which, just like the naive boundary term, ensures preservation of the variational principle under the conformal rescaling of $\S$ I.C. However, our improved boundary term is zweibein-gauge independent (in a sense to be made precise below). Therefore, as we shall explicitly demonstrate in the canonical formalism, our improved boundary term maintains complete zweibein-gauge freedom at the boundary. Moreover, as we will also show in the next section, our improved boundary term affords a rigorous correspondence between ECMs (zweibein theories) and GDTs (metric theories).

In order to write down the improved boundary term, we first collect a few definitions. Recall that we may write the general-gauge spacetime dual-zweibein as either (2.7) or (2.8), where the connection one-form associated with the arbitrary gauge is $\omega$. Define the time gauge by setting $\rho=0, \sigma_{L}=1$, and denote the connection 1-form for this special gauge by $\Omega=\omega+\mathrm{d} \rho$. Also, define the radial gauge by setting $\bar{\rho}=0, \sigma_{L}=1$, and denote the connection 1-form for this special gauge by $\bar{\Omega}=\omega+\mathrm{d} \bar{\rho}$. Clearly, as stated before in $\S$ II, the parameter associated with the local boost between the time-gauge and radial gauge zweibeine is $\eta=\bar{\rho}-\rho$. Our improved boundary term is

$$
L_{\partial \mathcal{M}}=-\kappa \oint_{\partial \mathcal{M}} X \omega+\kappa \int_{\Sigma^{\prime}}^{\Sigma^{\prime \prime}} \rho \mathrm{d} X-\kappa \int_{\overline{\mathcal{T}}} \bar{\rho} \mathrm{d} X
$$

where in this expression one should realize that $\rho$ and $\bar{\rho}$ are short-hand for $\frac{1}{2} \log \left(-e^{+}{ }_{r} / e^{-}{ }_{r}\right)$ and $\frac{1}{2} \log \left(e^{+}{ }_{t} / e^{-}{ }_{t}\right)$, respectively. Note that both $-e^{+}{ }_{r} / e^{-}{ }_{r}$ and $e^{+}{ }_{t} / e^{-}{ }_{t}$ are everywhere positive, so these logarithms make sense. Moreover, as both $\rho$ and $\bar{\rho}$ are conformally invariant, the extra two terms appearing on the right-hand side of (5.1) do not spoil the nice property that addition of (5.1) to the ECM action (1.1), like addition of the naive boundary term (1.2), ensures conformally preserved boundary conditions. In (5.1) and below we adopt the following convention for integration:

$$
\oint_{\partial \mathcal{M}}=\int_{\Sigma^{\prime}}^{\Sigma^{\prime \prime}}-\int_{\overline{\mathcal{T}}}+\left.\right|_{B^{\prime}} ^{B^{\prime \prime}}
$$

with

$$
\begin{gathered}
\int_{\Sigma^{\prime}}^{\Sigma^{\prime \prime}}=\int_{\Sigma^{\prime \prime}}-\int_{\Sigma^{\prime}} \text { and, for example, } \int_{\Sigma^{\prime}}=\int_{r_{i}}^{r_{o}} \text { at } t=t^{\prime} \\
\int_{\overline{\mathcal{T}}}=\int_{\overline{\mathcal{T}}_{o}}-\int_{\overline{\mathcal{T}}_{i}} \text { and, for example, } \int_{\overline{\mathcal{T}}_{i}}=\int_{t^{\prime}}^{t^{\prime \prime}} \text { at } r=r_{i} \\
\left.\right|_{B^{\prime}} ^{B^{\prime \prime}}=\left.\right|_{B_{o}^{\prime}} ^{B_{o}^{\prime \prime}}-\left.\right|_{B_{i}^{\prime}} ^{B_{i}^{\prime \prime}} .
\end{gathered}
$$

[These conventions ensure "clockwise" integration over the entire boundary $\partial \mathcal{M}$ for the integrals and corner terms in (5.5) below (see Fig. 1).] Because the corner points are a set of measure zero in $\partial \mathcal{M}$, the term $\left.\right|_{B^{\prime}} ^{B^{\prime \prime}}$ in $(5.2)$ is irrelevant unless the quantity being integrated over the boundary has distributional support at the corners. In particular, there are no corner-point contributions to $-\kappa \oint_{\partial \mathcal{M}} X \omega$ in (5.1).

To gain insight into the geometric meaning of the boundary term (5.1), on $\Sigma^{\prime}$ and $\Sigma^{\prime \prime}$ replace $\omega$ with $\Omega-\mathrm{d} \rho$, and on the $\overline{\mathcal{T}}$ boundary replace $\omega$ with $\bar{\Omega}-\mathrm{d} \bar{\rho}$. Next, integrate by 
parts to shift the d's off of the boost parameters $\rho$ and $\bar{\rho}$ and onto $X$ 's, thereby creating corner terms at $B_{o}^{\prime \prime}, B_{o}^{\prime}, B_{i}^{\prime \prime}$, and $B_{i}^{\prime}$. The result of this calculation is

$$
L_{\partial \mathcal{M}}=\kappa \int_{\Sigma^{\prime}}^{\Sigma^{\prime \prime}} \mathrm{d} r \Lambda X K-\kappa \int_{\overline{\mathcal{T}}} \mathrm{d} t \bar{N} X \bar{\Theta}-\left.\kappa X \eta\right|_{B^{\prime}} ^{B^{\prime \prime}}
$$

where, respectively, $K:=-\Omega[n]=-\nabla_{\mu} u^{\mu}$ and $\bar{\Theta}:=-\bar{\Omega}[\bar{u}]=-\nabla_{\mu} \bar{n}^{\mu}$ are by definition the mean curvatures associated with $\Sigma^{\prime \prime}$ (or $\Sigma^{\prime}$ ) as embedded in $\mathcal{M}$ and $\overline{\mathcal{T}}_{o}$ (or $\overline{\mathcal{T}}_{i}$ ) as embedded in $\mathcal{M}$. As before, the variable $\Lambda$ is the square root of the induced $\Sigma$ one-metric $\Lambda^{2}=-2 e^{+}{ }_{r} e^{-}{ }_{r}$, and, likewise, $\bar{N}$ is the square root of minus the induced $\overline{\mathcal{T}}$ one-metric $\bar{N}^{2}=2 e^{+}{ }_{t} e^{-}{ }_{t}$. Therefore, perhaps rather symbolically, we may write

$$
L_{\partial \mathcal{M}}=\kappa \oint_{\partial \mathcal{M}} \mathrm{d} x \sqrt{\left|g^{1}\right|} X \mathcal{K},
$$

with $\mathcal{K}$ denoting the mean curvature of the total boundary $\partial \mathcal{M}$ as embedded in $\mathcal{M}$ [in a generalized sense to the usual notion of mean curvature from (pseudo)Riemannian geometry, as $\nabla_{\mu}$ is the covariant derivative associated with a connection which may have torsion]. In other words, (5.1) is the " $X$-averaged" total mean curvature of the boundary as embedded in spacetime. The geometric importance of such a boundary term is well-known 36,23,24,37. In particular, such a term is zweibein-gauge independent, i. e. depends solely on metric variables (and here possibly torsion). Although the corner points constitute a set of measure zero in the integration of $X \mathcal{K}$ over the whole spacetime boundary, there are nevertheless finite contributions to the integral from these points as the $\partial \mathcal{M}$ normal changes discontinuously at these points, e. g. from $u^{\mu}$ to $\bar{n}^{\mu}$. Such corner terms are key in Brill and Hayward's treatment of the additivity of the 4d Einstein-gravity action under the composition of adjoining spacetime regions [37]. We note that our boundary term (5.1) differs from the type considered by Chan et al [19] mainly in that we take the corner terms explicitly into account.

Adding the boundary term (5.1) to the base part of the ECM action, we obtain

$$
L=\kappa \int_{\mathcal{M}}\left(-X_{a} D e^{a}+\omega \wedge \mathrm{d} X-V \epsilon\right)+\kappa \int_{\Sigma^{\prime}}^{\Sigma^{\prime \prime}} \rho \mathrm{d} X-\kappa \int_{\overline{\mathcal{T}}} \bar{\rho} \mathrm{d} X
$$

for the total action. (For simplicity, we ignore the matter contribution $L^{(m)}$ to the action here. As long as the matter is minimally coupled, its inclusion is straightforward.) By direct, if tedious, calculation we find the following for the variation of this action:

$$
\begin{aligned}
\delta L= & {[\text { terms giving the EOMs }(3.1)] } \\
& +\int_{\Sigma^{\prime}}^{\Sigma^{\prime \prime}} \mathrm{d} r\left(P_{+} \delta e^{+}{ }_{r}+P_{-} \delta e^{-}{ }_{r}+P_{X} \delta X\right) \\
& +\int_{\overline{\mathcal{T}}} \mathrm{d} t\left(\bar{\Pi}_{+} \delta e^{+}{ }_{t}+\bar{\Pi}_{-} \delta e^{-}{ }_{t}+\bar{\Pi}_{X} \delta X\right)-\left.\kappa \eta \delta X\right|_{B^{\prime}} ^{B^{\prime \prime}},
\end{aligned}
$$

where we have defined the momentaf [cf. the footnote just after Eq.(1.1) for a description of the notation]

\footnotetext{
${ }^{8}$ The momenta appearing in 5.8 can easily be rewritten in terms of components, for example,
} 


$$
\begin{aligned}
P_{ \pm} & =\kappa\left(X^{\mp} \mp n^{\mp} n[X]\right) \\
P_{X} & =-\kappa \Lambda(\omega[n]+n[\rho]) \\
\bar{\Pi}_{ \pm} & =-\kappa\left(X^{\mp} \mp \bar{u}^{\mp} \bar{u}[X]\right) \\
\bar{\Pi}_{X} & =\kappa \bar{N}(\omega[\bar{u}]+\bar{u}[\bar{\rho}]) .
\end{aligned}
$$

Here and in what follows, we make extensive use of the notation $n^{ \pm}:=n^{\mu} e^{ \pm}{ }_{\mu}$ (and similarly for $\bar{u}^{ \pm}, u^{ \pm}$, and $\bar{n}^{ \pm}$). Inspection of the variation (5.7) of the action shows that, with respect to a generic spacelike $\Sigma$ slice, $P_{ \pm}$and $P_{X}$ are the gravitational momenta respectively conjugate to $e^{ \pm}{ }_{r}$ and $X$. Likewise, $\bar{\Pi}_{ \pm}$and $\bar{\Pi}_{X}$ are conjugate to $e^{ \pm}{ }_{t}$ and $X$ respectively, where now conjugacy is defined with respect to the $\overline{\mathcal{T}}$ boundary. At least heuristically, $-\kappa \eta$ is the momentum conjugate to $X$ at the corners. Notice that the variational principle associated with the action (5.6) features fixation of $X$ at the corners, in harmony with the fact that $X$ is also fixed on $\Sigma^{\prime}, \Sigma^{\prime \prime}$, and the $\overline{\mathcal{T}}$ boundary. By construction, $-2 n^{+} n^{-}=1,2 \bar{u}^{+} \bar{u}^{-}=1$. Note also that the boost parameters above are in fact $\rho=\frac{1}{2} \log \left(-n^{+} / n^{-}\right)$and $\bar{\rho}=\frac{1}{2} \log \left(\bar{u}^{+} / \bar{u}^{-}\right)$.

The gravitational momenta $\left\{P_{ \pm}, P_{X}\right\}$ are not the standard ones usually associated with the canonical formulation of first-order ECMs [6,7] (the standard ECM canonical variables appear in the next section). Therefore, a few words are in order concerning their origin and geometric significance. First, $P_{X}$ is clearly the general-gauge expression for $-\kappa \Lambda \Omega[n]$. To gain insight into the geometric meanings of $P_{ \pm}$, insert the identities $\delta e^{ \pm}{ }_{r}=n^{ \pm}(\delta \Lambda \pm \Lambda \delta \rho)$ and $\delta e^{ \pm}{ }_{t}=\bar{u}^{ \pm}(\delta \bar{N} \pm \bar{N} \delta \bar{\rho})$ into the variation (5.7) in order to find

$$
\begin{aligned}
(\delta L)_{\partial \mathcal{M}}= & \int_{\Sigma^{\prime}}^{\Sigma^{\prime \prime}} \mathrm{d} r\left(P_{\Lambda} \delta \Lambda+P_{\rho} \delta \rho+P_{X} \delta X\right) \\
& +\int_{\overline{\mathcal{T}}} \mathrm{d} t\left(\bar{\Pi}_{\bar{N}} \delta \bar{N}+\bar{\Pi}_{\bar{\rho}} \delta \bar{\rho}+\bar{\Pi}_{X} \delta X\right)-\left.\kappa \eta \delta X\right|_{B^{\prime}} ^{B^{\prime \prime}},
\end{aligned}
$$

where the alternative momenta are the following:

$$
\begin{aligned}
P_{\Lambda} & =\kappa\left(X^{+} n^{-}+X^{-} n^{+}\right) \\
P_{\rho} & =\kappa \Lambda\left(n[X]-X^{+} n^{-}+X^{-} n^{+}\right) \\
\bar{\Pi}_{\bar{N}} & =-\kappa\left(X^{+} \bar{u}^{-}+X^{-} \bar{u}^{+}\right) \\
\bar{\Pi}_{\bar{\rho}} & =-\kappa \bar{N}\left(\bar{u}[X]-X^{+} \bar{u}^{-}+X^{-} \bar{u}^{+}\right) .
\end{aligned}
$$

With respect to a generic spacelike $\Sigma$ slice, the gravitational momentum $P_{\Lambda}$ is conjugate to $\Lambda$. Likewise, with respect to the $\overline{\mathcal{T}}$ boundary, the momentum $\bar{\Pi}_{\bar{N}}$ is conjugate to $\bar{N}$. As we shall see later, the expression for $-\bar{\Pi}_{\bar{N}}$ is closely related to the notion of quasilocal energy in the formalism. Notice that the EOM $(3.1 \mathrm{c})$ shows that both $P_{\rho}$ and $\bar{\Pi}_{\bar{\rho}}$ vanish onshell. Therefore, $\rho$ and $\bar{\rho}$ need not be held fixed at the boundary in the variational principle associated with the action (5.6), as the equation of motion (3.1. ) ensures that both $P_{\rho}$ and

$P_{+}=\kappa\left(X^{-}+\frac{1}{2} X^{\prime} / e^{+}{ }_{r}\right)$ and $P_{X}=-\kappa\left(\omega_{r}+\rho^{\prime}\right)$. In fact, to obtain the variation (5.7) of the action, it is easiest to work with the canonical form (5.14) of the action, with the understanding that the momenta are short-hand for the aforementioned component expressions. 
$\bar{\Pi}_{\bar{\rho}}$ vanish for arbitrary variations $\delta \rho$ and $\delta \bar{\rho}$ about a classical solution. Hence, for our choice (5.6) of action, the presence of the boundary $\partial \mathcal{M}$ does not break the zweibein-gauge freedom of the theory. In particular, this merely reflects the fact that $\rho$ does not represent a true dynamical degree of freedom. In terms of the alternative canonical momenta (5.10) we find that

$$
P_{ \pm}=-n^{\mp}\left(P_{\Lambda} \pm P_{\rho} / \Lambda\right) .
$$

Therefore, modulo $P_{\rho}$ which vanishes on-shell, both $P_{ \pm}$are closely related to $P_{\Lambda}$. We may consider the canonical pairs

$$
\left\{P_{ \pm}, e^{ \pm} ; P_{X}, X\right\}
$$

as $\Sigma$-adapted canonical variables $(\Sigma \mathrm{CVs})$ which are nevertheless expressed in a general zweibein gauge. We have obtained these $\Sigma$ CVs rather than the standard ones [5] [7], because our special boundary term (5.1) has enacted a canonical transformation. As we shall see, the $\Sigma$ CVs are the proper ones to use when examining the correspondence between ECMs and GDTs, although they are not ideal to take advantage of the underlying Poisson structure associated with ECMs. [5.66]

\section{B. Quasilocal Energy and Momentum}

Finding the canonical form of the action (5.6) is not difficult. The volume-integral piece of (5.6) is treated in the usual way; therefore, let us concentrate on how to handle the boundary terms. To begin with, convert the combined $\Sigma^{\prime}$ and $\Sigma^{\prime \prime}$ integrals into a volume integral, and then perform an integration by parts on the $\rho \dot{X}^{\prime}$ term in the volume integral in order to shift the radial derivative off of $\dot{X}$ and onto $\rho$, thereby creating another $\overline{\mathcal{T}}$ boundary integral. At the same time, the term $\dot{\rho}=\frac{1}{2} \partial_{t} \log \left(-e^{+}{ }_{r} / e^{-}{ }_{r}\right)$ is expanded. These simple manipulations yield

$$
\kappa \int_{\Sigma^{\prime}}^{\Sigma^{\prime \prime}} \rho \mathrm{d} X-\kappa \int_{\overline{\mathcal{T}}} \bar{\rho} \mathrm{d} X=\kappa \int_{\mathcal{M}} \mathrm{d}^{2} x\left(\frac{1}{2} X^{\prime} \dot{e}^{+}{ }_{r} / e^{+}{ }_{r}-\frac{1}{2} X^{\prime} \dot{e}^{-}{ }_{r} / e^{-}{ }_{r}-\rho^{\prime} \dot{X}\right)-\kappa \int_{\overline{\mathcal{T}}} \mathrm{d} t \eta \dot{X}
$$

From this result the canonical form of the action follows:

$$
L=\int_{\mathcal{M}} \mathrm{d}^{2} x\left(P_{a} \dot{e}^{a}{ }_{r}+P_{X} \dot{X}-e^{a}{ }_{t} G_{a}-\omega_{t} G\right)-\int_{\overline{\mathcal{T}}} \mathrm{d} t(\kappa \eta \dot{X}+\bar{N} \bar{E}),
$$

with

\footnotetext{
${ }^{9}$ For the particular case of SSGR, Guven and Ó Murchadha [30], have used what are essentially the gravitational constraints in this form (straightforwardly generalized to include matter) in an extensive examination of the spherically symmetric gravitational collapse of matter. This lightlike form of the constraints for SSGR had been earlier obtained by Malec and Ó Murchadha [38]. One of the main achievements of this work has been the derivation of certain bounds on the "optical scalars," essentially our $X^{ \pm}$, during the collapse scheme.
} 


$$
\begin{aligned}
G_{ \pm} / \kappa & =-\Lambda\left(n\left[X^{\mp}\right] \mp X^{\mp} \omega[n] \pm V n^{\mp}\right) \\
G / \kappa & =-\Lambda\left(n[X]-X^{+} n^{-}+X^{-} n^{+}\right) \\
\bar{E} / \kappa & =X^{+} \bar{u}^{-}+X^{-} \bar{u}^{+}
\end{aligned}
$$

It is, of course, a trivial exercise to express $G_{ \pm}$in terms of the canonical variables $\left\{P_{ \pm} ; e^{ \pm}{ }_{r} ; P_{X}, X\right\}$ but the resulting expressions are slightly more complicated and are not needed here. Do note, however, that the $\Sigma$ CVs are tailored to simplify $G=\Lambda\left(P_{-} n^{-}-P_{+} n^{+}\right)$. As is well-known [6, [], $\left\{G_{a}, G\right\}$ comprise a set of first-class constraints, with associated Lagrange multipliers $\left\{e^{a}{ }_{t}, \omega_{t}\right\}$. We would like to point out that the structure of the boundary term in the canonical action (5.14) precisely mimics the corresponding boundary term in the canonical action for full $4 \mathrm{~d}$ general relativity, in particular possessing a "kinetic term" with the parameter describing the boost between $u$ and $\bar{u}$ (slice and boundary frames) playing the role of a "momentum" 23,24.

We interpret the expression for $\bar{E}$ as the quasilocal energy (QLE) associated with the $\bar{u}^{\mu}$ observers at the $\overline{\mathcal{T}}$ boundary. It is a convenient and standard practice to associate a separate energy with each boundary point [10, with the expression for the energy at an inner boundary point being minus the expression for the energy at an outer boundary point. (We emphasize that, although the inner and outer boundary-point expressions for the energy differ by a sign, minus the value of the inner boundary-point energy is not equal to the value of outer boundary-point energy.) For instance, $\left.\bar{E}\right|_{B_{o}}$ is the energy associated with the outer boundary point. Put precisely, $\left.\bar{E}\right|_{B_{o}}$ is the energy, associated with $B_{o}$, of the gravitational fields (and possibly minimally coupled matter fields, if they are present) which "live" on a spacelike slice $\bar{\Sigma}$, where $\bar{\Sigma}$ has $\bar{u}^{\mu}$ as its timelike normal at the point $B_{o}=\partial \bar{\Sigma}$. Inspection of the alternative variation (5.9) of the action verifies the following important statement: When evaluated on solutions to the EOMs, the energy $\left.\bar{E}\right|_{B_{o}}$, for example, is minus the time rate of change of the classical action (or Hamilton-Jacobi principal function) with respect to a unit stretch in $\left.\bar{N}\right|_{\overline{\mathcal{T}}_{o}}$, where $\left.\bar{N}\right|_{\overline{\mathcal{T}}_{o}}$ controls the lapse of proper time between neighboring points on $\overline{\mathcal{T}}_{o}$. For definiteness in what follows, we shall confine ourselves to the outer boundary-point expression for the energy.

The QLE of the physical fields defined on the slice $\Sigma$ and associated with the $u^{\mu}$ observers at the boundary $B_{o}=\partial \Sigma$ is

$$
\left.E\right|_{B_{o}}=\kappa\left(X^{+} u^{-}+X^{-} u^{+}\right)
$$

(Henceforth, we shall suppress all $\left.\right|_{B_{o}}$ 's, with the understanding the we only deal with the outer boundary-point expression.) Now, any suitable expression for the $\Sigma$ energy should be expressible solely in terms of the canonical variables of $\Sigma$, and this energy satisfies this

\footnotetext{
${ }^{10}$ The terminology comes from SSGR, where this energy is associated with a round sphere, which is identified with a point in our $1+1$ spacetime region $\mathcal{M} . \bar{E}$ is certainly not an energy density like, for example, the component $T_{t}^{t}$ of the matter energy-momentum tensor.

${ }^{11}$ There is an equivalence class of such $\bar{\Sigma}$ 's (of course, technically $\partial \bar{\Sigma}$ is the union $B_{i} \cup B_{o}$ ).
} 
requirement. Indeed, using the first of the important geometric identities[2]

$$
\begin{aligned}
& X^{+} u^{-}+X^{-} u^{+}=X^{-} n^{+}-X^{+} n^{-} \\
& X^{+} n^{-}+X^{-} n^{+}=X^{-} u^{+}-X^{+} u^{-}
\end{aligned}
$$

we write

$$
E=\kappa\left(X^{-} n^{+}-X^{+} n^{-}\right)
$$

Again, we could easily write the $X^{ \pm}, n^{ \pm}$here in terms of the chosen canonical variables, but choose not to. Despite its zweibein-gauge invariance [cf. the footnote just before (5.17)], the QLE is clearly foliation-dependent, because the foliation choice determines $n^{\mu}$. We shall need a QLE expression for each point of $\Sigma$. Fixing the sign of the energy by interpreting each $\Sigma$ point as an outer-boundary point for a portion of $\Sigma$, we can use the expression (5.18) as given. Notice that, modulo the Lorentz constraint $G$, the QLE has the form

$$
\begin{aligned}
E & =-\kappa n[X] \\
& \uparrow \text { equality modulo } G \text { [or equivalently modulo the EOM }(3.1 \mathrm{c})] .
\end{aligned}
$$

Furthermore, this result immediately implies that the correct expression for QLE in the conformally rescaled spacetime, that is the physical energy, is

$$
\tilde{E}=\exp (\varphi) E
$$

and, therefore, the expression for QLE in GDTs is simply $\tilde{E}=-\kappa \tilde{n}[X]$. Again, as a vector field $\tilde{n}^{\mu}=\tilde{\Lambda}^{-1} \delta_{r}^{\mu}$, and in fact $\tilde{n}^{\mu}=\exp (\varphi) n^{\mu}$. Finally, we would like to point out that the on-shell expression (5.19) for the energy is in fact negative in an (exterior) type I region, as for the classical solutions we consider $X^{\prime}>0$ in such a region (and as a result the $\tilde{E}$ in (5.20) is also negative in a type I region). One obtains a positive energy expression only after referencing the energy as given against the groundstate energy of the theory. We address this issue in detail in $\S$ VI.C.

We also define

$$
J=-\kappa\left(X^{+} n^{-}+X^{-} n^{+}\right)
$$

and consider $J$, which is nothing but $-P_{\Lambda}$, to be a quasilocal local momentum. Trivially, $J$ depends only on $\Sigma$ canonical variables. Notice that on-shell $\left.J\right|_{\Sigma^{\prime \prime}}$, for instance, is minus the rate of change of the classical action with respect to a unit-stretch in $\left.\Lambda\right|_{\Sigma^{\prime \prime}}$, where $\left.\Lambda\right|_{\Sigma^{\prime \prime}}$ controls the lapse of proper radial distance between neighboring points of $\Sigma^{\prime \prime}$. Again, inspection of (5.9) verifies this statement. Next, with the geometric identity $(5.17 \mathrm{~b})$ and the EOM (3.1c), one sees that the on-shell expression for $J$ is

\footnotetext{
${ }^{12}$ Respectively, $\left.5.17 \mathrm{a}\right)$ and $(5.17 \mathrm{~b})$ can be written as the manifestly zweibein-gauge invariant expressions $-X^{a} u^{b} \eta_{a b}=X^{a} n^{b} \epsilon_{a b}$ and $-X^{a} n^{b} \eta_{a b}=X^{a} u^{b} \epsilon_{a b}$.
} 


$$
\begin{aligned}
J & =\kappa u[X] \\
& \uparrow \text { equality modulo the EOM (3.1c). }
\end{aligned}
$$

The conformally rescaled expression is $\tilde{J}=\exp (\varphi) J$, and, hence, the expression for quasilocal momentum in GDT is simply $\tilde{J}=\kappa \tilde{u}[X]$. We can also define a quasilocal momentum $\bar{J}$ for the observers in the $\overline{\mathcal{T}}$ boundary,

$$
\bar{J}=-\kappa\left(X^{+} \bar{n}^{-}+X^{-} \bar{n}^{+}\right) .
$$

Eqs. (2.6) imply the following boost relations between the "barred" and "unbarred" quasilocal energy and momentum:

$$
\begin{aligned}
& \bar{E}=\gamma E-v \gamma J \\
& \bar{J}=\gamma J-v \gamma E .
\end{aligned}
$$

Up to now, our results concerning quasilocal energy-momentum are unaffected by the inclusion of $2 \mathrm{~d}$ minimally coupled matter.

We now turn to the special case when the spacetimes under consideration possess an isometry (as is always true in vacuo). Just like before, we define quasilocal energy and momentum expressions for the preferred foliation $\hat{\Sigma}$ associated with the Killing direction, [cf. Eq. (2.10)]

$$
\begin{aligned}
\hat{E} & =\frac{1}{2} \kappa\left(\sigma_{+}+\sigma_{-}\right)\left(X^{-} \hat{n}^{+}-X^{+} \hat{n}^{-}\right)+\frac{1}{2} \kappa\left(\sigma_{+}-\sigma_{-}\right)\left(X^{-} \hat{u}^{+}-X^{+} \hat{u}^{-}\right) \\
-\hat{J} & =\frac{1}{2} \kappa\left(\sigma_{+}+\sigma_{-}\right)\left(X^{+} \hat{n}^{-}+X^{-} \hat{n}^{+}\right)+\frac{1}{2} \kappa\left(\sigma_{+}-\sigma_{-}\right)\left(X^{+} \hat{u}^{-}+X^{-} \hat{u}^{+}\right) .
\end{aligned}
$$

Notice the appearance of $\sigma_{ \pm}$in these expressions, which is necessary to cope with the fact that for the pathological $\hat{\Sigma}$ foliation the roles of $\sigma_{+} \hat{u}$ and $\sigma_{-} \hat{n}$ switch at the horizon. With the exception of the horizon where $\sigma_{K}$ is not defined, the relations (2.10) along with the identities $(5.17 \mathrm{a}, \mathrm{b})$ determine

$$
\begin{aligned}
\hat{E} & =\hat{\gamma} E-\hat{v} \hat{\gamma} J \\
\hat{J} & =\hat{\gamma} J-\hat{v} \hat{\gamma} E
\end{aligned}
$$

as the associated boost relations between "hatted" and "unhatted" quasilocal energy and momentum. Now, in fact, the "hatted" versions of the identities (5.17a,b) in tandem with the EOM (3.1 c) tell us that in a static region $\hat{J}=\kappa \sigma_{+} \hat{u}[X]=0$, while in a dynamical region $\hat{E}=-\kappa \sigma_{+} \hat{u}[X]=0$. The last equalities follow since $\hat{u}^{\mu}$ points along the Killing direction. Therefore, by the boost relations inverse to (5.26), we know that on-shell the rapidity boost parameter

$$
\begin{aligned}
\hat{\eta}=\frac{1}{2} \log \left|\frac{E+J}{E-J}\right| & =\frac{1}{2} \log \left|\frac{1+\hat{v}}{1-\hat{v}}\right| \\
& \uparrow \text { equality modulo the EOM (3.1c). }
\end{aligned}
$$

describes the local boost between $u^{\mu}$ and $\sigma_{+} \hat{u}^{\mu}$ (or $\sigma_{-} \hat{n}^{\mu}$ in dynamical region), that is between the $\Sigma$ and $\hat{\Sigma}$ frames. We have, therefore, an expression in terms of the $\Sigma$ canonical variables 
for the boost parameter introduced and discussed in $\S$ II (in the non-vacuum case it certainly may not be possible to obtain such an expression). A straightforward calculation shows that

$$
\hat{\eta}=-\frac{1}{2} \log \left(-n^{+} / n^{-}\right)+\frac{1}{2} \log \left|X^{+} / X^{-}\right| ;
$$

or, in other words, that $\xi=\rho+\hat{\eta}$. Here for later purposes we define a new boost parameter

$$
\xi:=\frac{1}{2} \log \left|X^{+} / X^{-}\right|,
$$

also to be called a rapidity. Clearly, the parameter $\xi$ is $\mp \infty$ if $X^{ \pm}=0$ vanishes while $X^{\mp}$ does not. Indeed, as is evident from the identity

$$
X^{+} / X^{-}=\sigma_{K} \exp (\alpha X)|C-W|^{-1}\left(X^{+}\right)^{2},
$$

this happens at the horizon. As one might expect, the limiting value of $\xi$, like $\hat{\eta}$, at a bifurcation point in a Kruskal-type diagram is direction-dependent.

\section{CANONICAL TRANSFORMATIONS AND REST-FRAME ENERGY}

In this section we have several goals in mind. First, we provide a rigorous derivation of GDT from the ECM formalism. Second, we obtain the preferred canonical variables (for both ECMs and GDTs) which are associated with the underlying Poisson structure (implicit in the ECMs we consider). The preferred canonical variables we obtain are essentially the "CasimirDarboux coordinates" used by Klösch and Strobl [6]. For reasons which will be evident, we call our preferred canonical variables "rapidity canonical variables" (RCVs). Third, having found the RCVs, we pass to the reduced phase space of the theory by elimination of the constraints, in order to obtain the "true degrees of freedom" for both (vacuum) ECMs and GDTs simultaneously. Fourth, we use the RCVs to examine the concept of "rest-frame" energy for GDTs with a Minkowski-spacetime ground state. Throughout this section we work in vacuo, although the derivation of GDT from the ECM formalism is unaffected by the inclusion of matter coupled in a not too "exotic" manner.

\section{A. Derivation of GDTs from ECMs}

As is evident from the alternative variation (5.9) of the action, the following is a trivial canonical transformation:

$$
\left\{P_{ \pm}, e^{ \pm}{ }_{r} P_{X}, X\right\} \rightarrow\left\{P_{\Lambda}, \Lambda ; P_{X}, X ; P_{\rho}, \rho\right\}
$$

By "trivial," we mean that the transformation requires no generating functional. Along with this canonical transformation, one should also recombine the constraints. (Although, depending on one's ultimate goal, this may be work done now which has to be undone later!)

The proper recombination is easily achieved by inserting the identities $e^{ \pm}{ }_{t}=\sqrt{\frac{1}{2}} \sigma_{L} e^{ \pm \rho}(N \pm$ $\Lambda N^{r}$ ) into the canonical action (5.14), with the result

$$
L=\int_{\mathcal{M}} \mathrm{d}^{2} x\left(P_{\Lambda} \dot{\Lambda}+P_{X} \dot{X}+P_{\rho} \dot{\rho}-N \mathcal{H}-N^{r} \mathcal{H}_{r}-\omega_{t} G\right)-\int_{\overline{\mathcal{T}}} \mathrm{d} t(\kappa \eta \dot{X}+\bar{N} \bar{E}),
$$


where $E$ and $J$ (found in $\bar{E}=\gamma E-v \gamma J$ ), $G$, and the new constraints

$$
\begin{aligned}
\mathcal{H} & =\sqrt{\frac{1}{2}} \sigma_{L}\left(e^{\rho} G_{+}+e^{-\rho} G_{-}\right) \\
\mathcal{H}_{r} & =\sqrt{\frac{1}{2}} \sigma_{L} \Lambda\left(e^{\rho} G_{+}-e^{-\rho} G_{-}\right)
\end{aligned}
$$

must be expressed in terms of $\left\{P_{\Lambda}, \Lambda ; P_{X}, X ; P_{\rho}, \rho\right\}$. In fact, as is evident from (5.9), one already has $P_{\rho}=-G$. A straightforward calculation yields the remaining set

$$
\begin{aligned}
\mathcal{H}= & -\kappa^{-1} P_{\Lambda} P_{X}+\left[\Lambda^{-1}\left(\kappa X^{\prime}-P_{\rho}\right)\right]^{\prime} \\
& +\frac{1}{2} \alpha \kappa^{-1}\left[\Lambda^{-1}\left(\kappa X^{\prime}-P_{\rho}\right)^{2}-\Lambda\left(P_{\Lambda}\right)^{2}\right]+\kappa \lambda^{2} \Lambda V_{0} \\
\mathcal{H}_{r}= & -\Lambda\left(P_{\Lambda}\right)^{\prime}+P_{X} X^{\prime}-\kappa^{-1} P_{X} P_{\rho} \\
\bar{E}= & -\gamma \Lambda^{-1}\left(\kappa X^{\prime}-P_{\rho}\right)+v \gamma P_{\Lambda}
\end{aligned}
$$

Since the pair $\left\{P_{\rho}, \rho\right\}$ is "pure gauge," it may be eliminated completely (set to zero in all equations) without affecting the solutions of the constraints and equations of motion for the remaining canonical variables. The elimination of this kinematic phase-space pair corresponds, at the covariant level, to solving the EOM (3.1. c) and substituting the solution back into the ECM action. We wish to stress that our ability to eliminate this gauge pair is intimately related to the particular boundary term (5.1) that we have added to the ECM action. (Of course, a similar elimination of this gauge pair would have been possible had we added no boundary integral at all to the ECM action; however, were we to follow that route, we would encounter an obstacle when trying to perform the "conformal" canonical transformation below.) The result of this elimination is a perfectly good canonical form of the theory based on $\left\{P_{\Lambda}, \Lambda ; P_{X}, X\right\}$.

Now, recall that, at the covariant level, a step in the passage from ECMs to GDTs involves the conformal rescaling discussed in $\S$ I.C [8]. At the canonical level this conformal rescaling is mirrored as a "trivial" canonical transformation subsequent ${ }^{13}$ to the first one (6.1),

$$
\left\{P_{\Lambda}, \Lambda ; P_{X}, X\right\} \rightarrow\left\{\mathcal{P}_{\tilde{\Lambda}}, \tilde{\Lambda} ; \mathcal{P}_{X}, X\right\}
$$

where the new canonical variables are

$$
\begin{aligned}
\mathcal{P}_{\tilde{\Lambda}} & =\exp (\varphi) P_{\Lambda} \\
\tilde{\Lambda} & =\exp (-\varphi) \Lambda \\
\mathcal{P}_{X} & =P_{X}+(\mathrm{d} \varphi / \mathrm{d} X) \Lambda P_{\Lambda} .
\end{aligned}
$$

Of course, the choice of conformal factor is model-dependent. Under this canonical transformation, we obtain the canonical action corresponding to a GDT, namely,

$$
\tilde{L}=\int_{\mathcal{M}} \mathrm{d}^{2} x\left(\mathcal{P}_{\tilde{\Lambda}} \dot{\tilde{\Lambda}}+\mathcal{P}_{X} \dot{X}-\tilde{N} \tilde{\mathcal{H}}-N^{r} \mathcal{H}_{r}\right)-\int_{\overline{\mathcal{T}}} \mathrm{d} t(\kappa \eta \dot{X}+\tilde{\bar{N}} \tilde{\bar{E}})
$$

\footnotetext{
${ }^{13}$ Actually, the "conformal" canonical transformation can be performed prior to the transformation (6.1) (and hence prior to elimination of the gauge pair $\left\{P_{\rho}, \rho\right\}$ ).
} 
with the following expressions for the constraints and quasilocal energy:

$$
\begin{aligned}
\tilde{\mathcal{H}}= & -\kappa^{-1} \mathcal{P}_{\tilde{\Lambda}} \mathcal{P}_{X}+\kappa^{-1}\left(\mathrm{~d} \varphi^{N E W} / \mathrm{d} X\right) \tilde{\Lambda}\left(\mathcal{P}_{\tilde{\Lambda}}\right)^{2} \\
& +\kappa \exp \left(\varphi^{N E W}\right)\left[\exp \left(-\varphi^{N E W}\right) \tilde{\Lambda}^{-1} X^{\prime}\right]^{\prime}+\kappa \lambda^{2} \exp (2 \varphi) \tilde{\Lambda} V_{0} \\
\mathcal{H}_{r}= & -\tilde{\Lambda}\left(\mathcal{P}_{\tilde{\Lambda}}\right)^{\prime}+\mathcal{P}_{X} X^{\prime} \\
\tilde{\bar{E}}= & -\gamma \tilde{\Lambda}^{-1} \kappa X^{\prime}+v \gamma \mathcal{P}_{\tilde{\Lambda}} .
\end{aligned}
$$

Here we have defined the "new" conformal factor"

$$
\varphi^{N E W}:=\varphi-\frac{1}{2} \int_{X_{0}}^{X} \mathrm{~d} y \alpha(y)
$$

by subtracting a field integral of $\alpha$ from the "old" conformal factor. Notice that the lapse $\tilde{N}$, boundary lapse $\tilde{\bar{N}}$, Hamiltonian constraint $\tilde{\mathcal{H}}$, and quasilocal energy $\tilde{\bar{E}}=\gamma \tilde{E}-v \gamma \tilde{J}$ are rescaled quantities, while the shift $N^{r}$ and momentum constraint $\mathcal{H}_{r}$ are not (although, of course, $\mathcal{H}_{r}$ must still be re-expressed in terms of the new "tilded" canonical variables).

The ability to perform the canonical transformation (6.5) renders the function $\alpha$ locally irrelevant (although vastly different global properties can stem from changes in $\alpha$ and from the conformal rescaling [8]!). Indeed, because $V_{0}=V_{0}(X)$ is essentially arbitrary in our formalism, we may rewrite the potential term $V_{0}$ in $\tilde{\mathcal{H}}$ as

$$
V_{0}=\exp \left[-\int_{X_{0}}^{X} \mathrm{~d} y \alpha(y)\right] V_{0}^{N E W} .
$$

Then, we can substitute $\exp \left(2 \varphi^{N E W}\right) V_{0}^{N E W}$ for $\exp (2 \varphi) V_{0}$ in the right-most term in (6.8 a). This shows that $\tilde{\mathcal{H}}$ may be obtained from $\mathcal{H}$ above with $\alpha=0$ by using the new conformal factor (6.9) and choosing a slightly different potential $V_{0}$. This is our argument as to why our choice of $\alpha=0$ in $\S \mathrm{IV}$ results in no loss of generality at the level of GDT as long as $\mathcal{M}$ is not "cut" but the conformal transformation."ㅁㅁ Finally, if desired, we may make a yet another trivial canonical transformation in order to trade the pair $\left\{\mathcal{P}_{X}, X\right\}$ for the new pair $\left\{\mathcal{P}_{\tilde{X}}, \tilde{X}\right\}$, with $\tilde{X}$ the dilaton field of $\S$ IV and $\mathcal{P}_{\tilde{X}}=2 \lambda^{-2}\left|W^{N E W}\right| \mathcal{P}_{X}$ (here $W^{N E W}$ has the same definition as $W$ but with $V_{0}^{N E W}$ ). The result of our labors is the canonical form of the action (4.4) [provided, of course, that in (4.4) the zweibein as been suitably gauge-fixed at the boundary so that (4.4) is truly a metric action].

For the special cases of SSGR and 2dDG in the presence of a Killing field, the base piece of the canonical ADM action (6.7) above is the starting point for many authors [4, 22, 20, 39, 40], who then essentially work in a direction opposite to our course in this subsection with the ultimate goal of obtaining the "true degrees of freedom," via a canonical transformation of

\footnotetext{
${ }^{14}$ Here, for once, we have dropped our previous restriction to $\alpha=$ const. in (1.3), i. e. we allow for the general case $\alpha=\alpha(X)$.

${ }^{15} \mathrm{~A}$ positive (or negative) conformal factor typically produces new geodesically complete edges inside the global Penrose diagram of the corresponding ECM [8].
} 
the form $\left\{\mathcal{P}_{\tilde{\Lambda}}, \tilde{\Lambda} ; \mathcal{P}_{X}, X\right\} \rightarrow\left\{\mathrm{P}_{C}, C ; \mathrm{P}_{X}, X\right\}$. Again $C$ is our absolutely conserved quantity which corresponds in metric-variable SSGR to Kuchař's canonical expression for the SBH mass parameter [ []. Such a transformation and mass canonical variable have also been considered for GDT in Refs. [35]. In the next subsection we shall obtain the pair $\left\{\mathrm{P}_{C}, C ; \mathrm{P}_{X}, X\right\}$ in a more direct fashion from the ECM canonical variables.

\section{B. Rapidity Canonical Variables}

We now perform two successive canonical transformations on the $\Sigma \mathrm{CVs}\left\{P_{a}, e^{a}{ }_{r} ; P_{X}, X\right\}$ in order to obtain the RCVs which clearly exhibit the true degrees of freedom for the models we study. Also examined in detail is the generating functional for the composite transformation. The first canonical transformation $\left\{P_{a}, e^{a}{ }_{r} ; P_{X}, X\right\} \rightarrow\left\{\mathcal{P}_{a}, e^{a}{ }_{r} ; \mathcal{P}_{X}, X\right\}$ is to the standard (Poisson-sigma model) canonical variables of an ECM: [5] 7] $\mathcal{P}_{ \pm}=\kappa X^{\mp}$ and $\mathcal{P}_{X}=-\kappa \Lambda \omega[n]$. That this transformation is indeed canonical is verified by the identity

$$
P_{a} \delta e_{r}^{a}+P_{X} \delta X-\mathcal{P}_{a} \delta e^{a}{ }_{r}-\mathcal{P}_{X} \delta X=\kappa \delta\left(\rho X^{\prime}\right)-\kappa(\rho \delta X)^{\prime}
$$

which upon integration over a generic slice $\Sigma$ shows that, for our boundary conditions (that is, fixation of $X$ ), the difference between the old and new Liouville forms is an exact form. Hence, the transformation is canonical. We now perform a second canonical transformation to rapidity canonical variables [so-named since the rapidity variable (5.29) appears as a

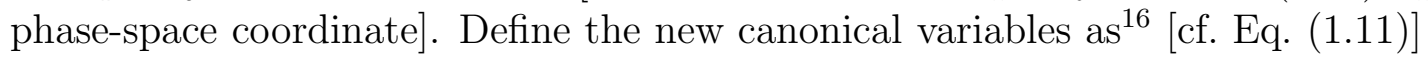

$$
\begin{aligned}
C & =\exp (\alpha X) X^{+} X^{-}+W \\
\mathrm{P}_{C} & =-\frac{1}{2} \kappa \Lambda \exp (-\alpha X)\left[\left(n^{+} / X^{+}\right)+\left(n^{-} / X^{-}\right)\right] \\
\xi & =\frac{1}{2} \log \left|X^{+} / X^{-}\right| \\
\mathrm{P}_{\xi} & =\kappa \Lambda\left(n[X]-n^{-} X^{+}+n^{+} X^{-}\right) \\
X & =X \\
\mathrm{P}_{X} & =-\kappa \Lambda\left\{\omega[n]-\frac{1}{2} V\left[\left(n^{+} / X^{+}\right)+\left(n^{-} / X^{-}\right)\right]+\frac{1}{2} n\left[\log \left|X^{+} / X^{-}\right|\right]\right\} .
\end{aligned}
$$

Clearly, this transformation breaks down on a horizon. However, for the time being, we shall ignore this fact. Note that $\mathrm{P}_{\xi}=-G$; and, therefore, one expects the pair $\left\{\mathrm{P}_{\xi}, \xi\right\}$ to be "pure gauge." This expectation turns out to be correct. A comparison with the approach of Klösch and Strobl [6] is in order. They work with coordinates in which only $\log \left|X^{+}\right|$ (or $\log \left|X^{-}\right|$, but not the logarithm of the ratio) and its conjugate appear as one of the phase-space pairs. This has advantages and disadvantages. The former being that a given set of Klösch and Strobl's canonical variables are good on part of the horizon, in marked

\footnotetext{
${ }^{16} \mathrm{In}(6.12)$ and some of the equations to follow, the purist will wish to express $X^{ \pm}, \Lambda n^{ \pm}, \Lambda \omega[n]$, and $\Lambda n[X]$ in terms of either $\left\{\mathcal{P}_{a}, e^{a}{ }_{r}, \mathcal{P}_{X}, X\right\}$ or, as the case may be, the original canonical variables $\left\{P_{a}, e_{r}^{a} ; P_{X}, X\right\}$; however, as mentioned, in order to keep the expressions as simple as possible, we shall not do so.
} 
contrast to our variables which are bad on the whole horizon. However, our RCVs afford easy passage to metric GDT.

To establish that the composite transformation $\left\{P_{ \pm}, e^{ \pm}{ }_{r} ; P_{X}, X\right\} \rightarrow\left\{\mathrm{P}_{C}, C ; \mathrm{P}_{X}, X ; \mathrm{P}_{\xi}, \xi\right\}$ from $\Sigma$ CVs to RCVs is canonical, first verify the following identity:

$$
\mathcal{P}_{a} \delta e^{a}{ }_{r}+\mathcal{P}_{X} \delta X-\mathrm{P}_{C} \delta C-\mathrm{P}_{\xi} \delta \xi-\mathrm{P}_{X} \delta X=-\kappa \delta\left(X_{a} e^{a}{ }_{r}+\xi X^{\prime}\right)+\kappa(\xi \delta X)^{\prime}
$$

which when combined with (6.11) gives a third identity

$$
P_{a} \delta e^{a}{ }_{r}+P_{X} \delta X-\mathrm{P}_{C} \delta C-\mathrm{P}_{\xi} \delta \xi-\mathrm{P}_{X} \delta X=-\kappa \delta\left[X_{a} e^{a}{ }_{r}+(\xi-\rho) X^{\prime}\right]+\kappa[(\xi-\rho) \delta X]^{\prime} .
$$

Integration of (6.14) over a generic slice $\Sigma$ yields

$$
\begin{aligned}
& \int_{\Sigma} \mathrm{d} r\left(P_{a} \delta e^{a}{ }_{r}+P_{X} \delta X\right)-\int_{\Sigma} \mathrm{d} r\left(\mathrm{P}_{C} \delta C+\mathrm{P}_{\xi} \delta \xi+\mathrm{P}_{X} \delta X\right)= \\
& -\kappa \delta \int_{\Sigma} \mathrm{d} r\left[X_{a} e^{a}{ }_{r}+(\xi-\rho) X^{\prime}\right]+\left.\kappa[(\xi-\rho) \delta X]\right|_{B_{i}} ^{B_{o}}
\end{aligned}
$$

therefore, for our boundary conditions (that is, with fixation of $X$ at the boundary $\partial \Sigma=$ $B_{i} \cup B_{o}$ ) the difference between the old and new Liouville forms is an exact form. Hence, the composite transformation is indeed canonical, although to be performed it requires a generating functional, namely,

$$
\left.\Xi\right|_{\Sigma}:=\kappa \int_{\Sigma} \mathrm{d} r\left[X_{a} e^{a}+(\xi-\rho) X^{\prime}\right]
$$

Using the results of $\S \mathrm{V}$, we easily see that the generating functional depends on the quasilocal energy and momentum,

$$
\left.\Xi\right|_{\Sigma}=\int_{\Sigma} \mathrm{d} r \Lambda[J-\hat{\eta} E-\hat{\eta} G / \Lambda]
$$

We could, of course, work with a slightly modified generating functional

$$
\left.\Upsilon\right|_{\Sigma}:=\int_{\Sigma} \mathrm{d} r \Lambda[J-\hat{\eta} E]
$$

in place of $\left.\Xi\right|_{\Sigma}$. This is permissible, as using $\Upsilon$ amounts to the addition of an EOM boundary term to the action, i. e. a term which vanishes on-shell. In this case, if one is willing to redefine the Lagrange parameter associated with the Lorentz constraint, then $\left.\Upsilon\right|_{\Sigma}$ gives rise to slightly different new canonical variables, namely, $\left\{\mathrm{P}_{C}, C ; \mathrm{P}_{X}, X ; \mathrm{P}_{\rho}=\mathrm{P}_{\xi}, \rho\right\}$. But, so far we see no particular advantage to be gained by working with $\left.\Upsilon\right|_{\Sigma}$ in place of $\left.\Xi\right|_{\Sigma}$.

By replacing the arbitrary variation $\delta$ in (6.15) with a time derivative and then integrating the whole equation over time, we find

$$
\int_{\mathcal{M}} \mathrm{d}^{2} x\left(P_{a} \dot{e}^{a}{ }_{r}+P_{X} \dot{X}\right)+\left.\Xi\right|_{\Sigma^{\prime}} ^{\Sigma^{\prime \prime}}=\int_{\mathcal{M}} \mathrm{d}^{2} x\left(\mathrm{P}_{C} \dot{C}+\mathrm{P}_{X} \dot{X}+\mathrm{P}_{\xi} \dot{\xi}\right)+\int_{\overline{\mathcal{T}}} \mathrm{d} t \kappa \hat{\eta} \dot{X}
$$


Hence, defining a new action $L_{\dagger}=L+\left.\Xi\right|_{\Sigma^{\prime}} ^{\Sigma^{\prime \prime}}$, with $(\underline{6.19})$ one arrives at

$$
L_{\dagger}=\int_{\mathcal{M}} \mathrm{d}^{2} x\left(\mathrm{P}_{C} \dot{C}+\mathrm{P}_{X} \dot{X}+\mathrm{P}_{\xi} \dot{\xi}-e^{a}{ }_{t} G_{a}-\omega_{t} G\right)-\int_{\overline{\mathcal{T}}} \mathrm{d} t[\kappa(\eta-\hat{\eta}) \dot{X}+\bar{N} \bar{E}]
$$

for the canonical form of $L_{\dagger}$ [cf. with the action given in Eq. (5.14)]. Let us express the constraints and quasilocal energy in (6.20) in terms of the new canonical variables. It is trivial to obtain the new expression for the Lorentz constraint; however, one must exercise due caution with the signs of $\sigma_{ \pm}, \sigma_{K}$ when obtaining the new expressions for $G_{ \pm}$. Checking the identities

$$
\begin{aligned}
-\kappa C^{\prime} & =\exp (\alpha X)\left(X^{a} G_{a}+V G\right) \\
\mathrm{P}_{X} & =\frac{1}{2}\left[\left(G_{-} / X^{+}\right)-\left(G_{+} / X^{-}\right)\right]
\end{aligned}
$$

is straightforward. Indeed, the first is by now a well-known result [34, 41, 31, 6, 7]. From (6.21 a) and (6.12c) we determine that

$$
G_{ \pm}+\sigma_{K} e^{\mp 2 \xi} G_{\mp}=\left(X^{ \pm}\right)^{-1}\left[V \mathrm{P}_{\xi}-\kappa \exp (-\alpha X) C^{\prime}\right]
$$

where along the way we have written $X^{+} / X^{-}=\sigma_{K}\left|X^{+} / X^{-}\right|[$cf. after (5.30)] and have used $\mathrm{P}_{\xi}=-G$. Likewise, from $(6.21 \mathrm{~b})$ we find

$$
G_{ \pm}-\sigma_{K} e^{\mp 2 \xi} G_{\mp}=\mp X^{\mp} \mathrm{P}_{X}
$$

With previous two equations and $(6.12 \mathrm{a})$ we obtain the following new expressions for the constraints:

$$
\begin{aligned}
G_{ \pm} & =\Delta_{ \pm}\left[V \mathrm{P}_{\xi}-\kappa \exp (-\alpha X) C^{\prime} \mp 2 \exp (-\alpha X)(C-W) \mathrm{P}_{X}\right] \\
G & =-\mathrm{P}_{\xi}
\end{aligned}
$$

where the pre-factors are given by [cf. Eq. (5.30)]

$$
\Delta_{ \pm}:=\frac{1}{2}\left(X^{ \pm}\right)^{-1}=-\sigma_{ \pm} \sigma_{L} \frac{\exp \left(\frac{1}{2} \alpha X \mp \xi\right)}{\sqrt{4|C-w|}}
$$

with $\sigma_{ \pm}$determined by $X^{ \pm}=-\sigma_{ \pm} \sigma_{L}\left|X^{ \pm}\right|$as before in $\S$ II. From (3.5) and (6.12a), it follows that $\sigma_{+} \sigma_{-}=\sigma_{K}$, also as we have seen before. The expression for $\bar{E}$ in (6.20) is again $\gamma E-v \gamma J$, but with the expressions for $E$ and $J$ given in terms of the new variables (6.12). One finds the new expressions to be

$$
\begin{gathered}
E=-\Lambda^{-1} \kappa\left(\kappa X^{\prime}-P_{\xi}\right) \\
J=2 \Lambda^{-1} \mathrm{P}_{C}(C-W),
\end{gathered}
$$

\footnotetext{
${ }^{17}$ In Ref. [20] a step essentially identical to this one is handled poorly in obtaining equation (4.24) of that reference. Nevertheless, a heuristic argument in the text just after this equation compensates for this poor handling; hence, the analysis in that reference is correct.
} 
where here one should consider $\Lambda$ as short-hand for

$$
\Lambda=\kappa^{-1} \exp \left(\frac{1}{2} \alpha X\right)|C-W|^{-1 / 2}\left[\left|\kappa^{2}\left(X^{\prime}\right)^{2}-4\left(\mathrm{P}_{C}\right)^{2}(C-W)^{2}+\left(\mathrm{P}_{\xi}\right)^{2}-2 \kappa X^{\prime} \mathrm{P}_{\xi}\right|\right]^{1 / 2} .
$$

The derivation of this expression for $\Lambda$ requires inversion of the canonical transformation (6.12). We shall not need the expressions (6.26) and (6.27). We have collected these equations only to make the following two points: (i) there is a $\mathrm{P}_{C}$ "buried" in the new variable expression for $\bar{E}$ (coming from the new-variable expression for $\Lambda$ ); and (ii) the variable $\xi$ appears nowhere in the action $L_{\dagger}$ except in the kinetic term. Our point (ii) implies that the equation of motion for $\xi$ 's conjugate is $\dot{\mathrm{P}}_{\xi}=0$ (of course, $-\mathrm{P}_{\xi}$ is the Lorentz constraint!); therefore, as expected, that the pair $\left\{\mathrm{P}_{\xi}, \xi\right\}$ is indeed "pure-gauge."

\section{Canonical reduction}

In this subsection we turn to the canonical reduction of the (vacuum) ECMs and GDTs we study in this paper. That is to say, we shall solve all of the constraints and substitute their solutions back into the action, thereby obtaining a reduced action for the "true degrees of freedom." Moreover, the variational principle associated with our reduced action will feature fixation of the same relevant geometric quantities on the boundary as those which are fixed in the original action principle.

Inspection of (6.24) shows that the vanishing of the set $\left\{\mathrm{P}_{X}, \mathrm{P}_{\xi}, C^{\prime}\right\}$ is completely equivalent to the vanishing of the constraints. Therefore, let us re-express the action (6.20) as

$$
L_{\dagger}=\int_{\mathcal{M}} \mathrm{d}^{2} x\left(\mathrm{P}_{C} \dot{C}+\mathrm{P}_{X} \dot{X}+\mathrm{P}_{\xi} \dot{\xi}-N^{C} C^{\prime}-N^{X} \mathrm{P}_{X}-N^{\xi} \mathrm{P}_{\xi}\right)-\int_{\overline{\mathcal{T}}} \mathrm{d} t[\kappa(\eta-\hat{\eta}) \dot{X}+\bar{N} \bar{E}],
$$

with

$$
\begin{aligned}
N^{C} & =-\kappa \exp (-\alpha X)\left(\Delta_{+} e^{+}{ }_{t}+\Delta_{-} e^{-}{ }_{t}\right) \\
N^{X} & =2 \exp (-\alpha X)(C-W)\left(\Delta_{-} e^{-}{ }_{t}-\Delta_{+} e^{+}{ }_{t}\right) \\
N^{\xi} & =\omega_{t}-\kappa V\left(\Delta_{+} e^{+}{ }_{t}+\Delta_{-} e^{-}{ }_{t}\right) .
\end{aligned}
$$

The next step is to reinterpret the expressions (6.29) as freely variable Lagrange multipliers. However, as taking this step requires extreme caution, let us first examine the geometric interpretation of these multipliers in terms of the old variables. Using the definitions of $\bar{E}$ and $\bar{J},(6.12 \mathrm{a}),(6.25)$, and the identities (5.17a,b), we find that

$$
\begin{aligned}
& N^{C}=-\frac{1}{2}(C-W)^{-1} \bar{N} \bar{E} \\
& N^{X}=\kappa^{-1} \bar{N} \bar{J} .
\end{aligned}
$$

Note that $(6.30 \mathrm{~b})$ implies the on-shell equality $N^{X}=\dot{X}$. We shall find these expressions useful even after we have assumed that $N^{C}$ and $N^{X}$ are freely variable, an assumption which requires careful handling of the boundary terms in the action.

One of the new constraints is $C^{\prime}$; therefore, upon elimination of this constraint one expects the emergence of a new canonical pair $\left\{\mathbf{P}_{\mathbf{C}}, \mathbf{C}\right\}$ defined by $\mathbf{C}(t):=C(t)$ (the remaining 
"zero-mode" of $C$ ) and $\mathbf{P}_{\mathbf{C}}:=\int_{\Sigma} \mathrm{d} r \mathrm{P}_{C}$. Moreover, one would like to express the reduced action directly in terms of $\left\{\mathbf{P}_{\mathbf{C}}, \mathbf{C}\right\}$. However, note that our point (i) made after (6.27) would seem to obstruct the fulfillment of this wish, as the momentum $\mathrm{P}_{C}$ lies in boundary term explicitly. To bypass this obstruction and obtain the "correct" new action principle with freely variable $N^{C}, N^{X}$, and $N^{\xi}$, we shall follow the prescription explained in Refs. 22, 20] (which examined only cases of vacuum SSGR and 2dDG). Let us first go through the prescription to obtain the new action, and only afterwards comment on why this new action principle is the "correct" one.

The first ingredient we need is the identity

$$
\bar{N}^{2}=\exp (\alpha X)\left[2 \kappa^{-2}(C-W)\left(N^{C}\right)^{2}-\frac{1}{2}(C-W)^{-1}\left(N^{X}\right)^{2}\right],
$$

which may be verified by explicit calculation with (6.29a,b). Henceforth, the boldface symbol $\overline{\boldsymbol{N}}$ shall represent the square root of the right-hand side of (6.31). Next, using (5.18) and (5.21), we find that

$$
E^{2}-J^{2}=2 \kappa^{2} X^{+} X^{-}
$$

As is evident, the "boost invariant" $E^{2}-J^{2}$ is positive in static regions and negative in dynamical ones. By the relations (5.24), we know that $E^{2}-J^{2}=\bar{E}^{2}-\bar{J}^{2}$; and, therefore, using (6.12a), we have

$$
\bar{E}^{2}=2 \kappa^{2} \exp (-\alpha X)(C-W)+\bar{J}^{2}
$$

At this point, we use the EOM (3.1 b) and the definition (5.23) to define

$$
\overline{\boldsymbol{J}}:=\kappa \dot{X} / \overline{\mathbf{N}}
$$

which agrees with $\bar{J}$ on-shell. It now follows that, also on-shell, the expression we have considered for $\bar{E}$ is equivalent to

$$
\overline{\boldsymbol{E}}:=-\kappa \sqrt{2 \exp (-\alpha X)(C-W)+(\dot{X} / \overline{\boldsymbol{N}})^{2}}
$$

Notice that we have taken the negative square root. In fact, this is the appropriate sign choice for the (outer boundary-point) energy corresponding to our type I region [analogous to the (exterior) region I of the Penrose diagram for the $\mathrm{SBH}]$. The positive square root is the appropriate choice for the (outer boundary-point) energy expression corresponding to a type III region in the same example. ${ }^{18}$ In type II and IV regions the sign of the energy expression need not be everywhere the same (and, therefore, can be zero). The prescription we shall follow seems to be well-suited only when the energy expression takes a definite sign, i. e. within the $\overline{\mathcal{T}}$ boundary lies entirely within static regions. There are two possibilities: (i) both the timelike boundary elements $\overline{\mathcal{T}}_{i}$ and $\overline{\mathcal{T}}_{o}$ lie within the same static region (or, in

\footnotetext{
${ }^{18}$ In effect, the roles of "outer" and "inner" are reversed in a type III region. We have assumed that our coordinate $r$ increases monotonically from right to left in a Kruskal-type diagram.
} 
other words, the $\Sigma$ slices nowhere cut across the horizon), and (ii) the boundary elements $\bar{T}_{i}$ and $\overline{\mathcal{T}}_{o}$ lie in separate static regions (so that the $\Sigma$ slices do cut across the horizon and penetrate dynamical regions). Of course, possibility (ii) is perhaps the more interesting one, as it allows one to study foliations which sample larger portions of the maximal extension of $g_{\mu \nu}$. However, due caution is required when such a possibility is considered, as the canonical variables (6.12) would seem to be ill-defined at the horizon. For the case of SSGR Kuchar has addressed in detail the situation in which the $\Sigma$ slices do cut completely across the horizon [ []. Nevertheless, it is our understanding that with the canonical variables (6.12) this situation is not completely understood; hence, we shall steer clear of this issue. Besides our goal is to obtain an expression for the rest-frame energy, and for this goal the above possibility (i) suffices. Therefore, for the rest of the paper, we shall assume that the patch $\mathcal{M}$ lies entirely within a single static region (which for the sake of definiteness we take to be a type I region).

The parameter $\eta-\hat{\eta}$ describes the boost between the boundary $\bar{\Sigma}$ and Killing $\hat{\Sigma}$ frames. Therefore, we can combine the boost relations (5.24) and (5.26) in order to show that

$$
\begin{aligned}
& \qquad \eta-\hat{\eta}=\boldsymbol{\eta}:=-\frac{1}{2} \log \left|\frac{\overline{\boldsymbol{E}}+\overline{\boldsymbol{J}}}{\overline{\boldsymbol{E}}-\overline{\boldsymbol{J}}}\right| . \\
& \text { on }- \text { shell equality } \uparrow
\end{aligned}
$$

We now use the new expressions (6.35) and (6.34) to define a slightly different version of the action (6.28),

$$
L_{\ddagger}=\int_{\mathcal{M}} \mathrm{d}^{2} x\left(\mathrm{P}_{C} \dot{C}+\mathrm{P}_{X} \dot{X}+\mathrm{P}_{\xi} \dot{\xi}-N^{C} C^{\prime}-N^{X} \mathrm{P}_{X}-N^{\xi} \mathrm{P}_{\xi}\right)-\int_{\overline{\mathcal{T}}} \mathrm{d} t(\kappa \boldsymbol{\eta} \dot{X}+\overline{\boldsymbol{N}} \overline{\boldsymbol{E}}) .
$$

With $L_{\ddagger}$ we assume that the new Lagrange multipliers are freely variable. Let us verify that the new action $L_{\ddagger}$ possesses essentially the same variational principle as the original one $L$ from (5.14). First, it is easy to find the constraints and canonical equations of motion associated with $L_{\ddagger}$. These are the following:

$$
\begin{aligned}
C^{\prime} & =0 \\
\mathrm{P}_{X} & =0 \\
\mathrm{P}_{\xi} & =0 \\
\dot{\mathrm{P}}_{C} & =\left(N^{C}\right)^{\prime} \\
\dot{C} & =0 \\
\dot{\mathrm{P}}_{X} & =0 \\
\dot{X} & =N^{X} \\
\dot{\mathrm{P}}_{\xi} & =0 \\
\dot{\xi} & =N^{\xi} .
\end{aligned}
$$

Recall that with $(6.30 \mathrm{~b})$ we have already found $6.39 \mathrm{~g})$ to be a valid equation on-shell. Notice that both $\left\{\mathrm{P}_{X}, X\right\}$ and $\left\{\mathrm{P}_{\xi}, \xi\right\}$ are "pure-gauge" pairs.

The next step is to examine the boundary terms $\left(\delta L_{\ddagger}\right)_{\partial \mathcal{M}}$ in the variation $\delta L_{\ddagger}$ of the new action. By a tedious but straightforward calculation we find 


$$
\begin{aligned}
\left(\delta L_{\ddagger}\right)_{\partial \mathcal{M}}= & \int_{\Sigma^{\prime}}^{\Sigma^{\prime \prime}} \mathrm{d} r\left(\mathrm{P}_{C} \delta C+\mathrm{P}_{X} \delta X+\mathrm{P}_{\xi} \delta \xi\right) \\
& +\int_{\overline{\mathcal{T}}} \mathrm{d} t\left(\overline{\boldsymbol{\Pi}}_{\bar{N}} \delta \overline{\boldsymbol{N}}+\overline{\boldsymbol{\Pi}}_{C} \delta C+\overline{\boldsymbol{\Pi}}_{X} \delta X\right)-\left.\kappa \boldsymbol{\eta} \delta X\right|_{B^{\prime}} ^{B^{\prime \prime}},
\end{aligned}
$$

with the following new $\overline{\mathcal{T}}$ momenta:

$$
\begin{aligned}
\overline{\boldsymbol{\Pi}}_{\bar{N}} & =-\overline{\boldsymbol{E}} \\
\overline{\boldsymbol{\Pi}}_{X} & =\overline{\boldsymbol{N}}\left\{\frac{1}{2} \overline{\boldsymbol{E}}\left[\alpha+(C-W)^{-1} \lambda^{2} V_{0}\right]+\bar{u}[\boldsymbol{\eta}]\right\} \\
\overline{\boldsymbol{\Pi}}_{C} & =-\frac{1}{2}(C-W)^{-1} \overline{\boldsymbol{N}} \overline{\boldsymbol{E}}-N^{C} .
\end{aligned}
$$

Of course, the momentum $\bar{\Pi}_{\bar{N}}$ is not quite the momentum $\bar{\Pi}_{\bar{N}}$ considered in (5.10c), but it does agree with $\bar{\Pi}_{\bar{N}}$ on-shell. For positive $N^{C}$ the momenta $\bar{\Pi}_{C}$ vanishes on-shell. This can be shown by inserting into $\overline{\boldsymbol{\Pi}}_{C}$ the full expression (6.31) for $\overline{\boldsymbol{N}}^{2}$, using the canonical equation of motion $(6.39 \mathrm{~g})$, and realizing that $(C-W)$ is positive in a static region as we have here. Moreover, it is natural to assume that $N^{C}$ is positive, because as a canonical expression in terms of the old variables $C^{\prime}$ generates evolution forward in time along orbits of the isometry in our type I region of interest [29. [- 6]. Alternatively, one can "cheat" and use the interpretation $(6.30 \mathrm{a})$ to establish the result. Therefore, $C$ need not be fixed on the $\overline{\mathcal{T}}$ boundary in the variational principle associated with $L_{\ddagger}$, as the equations of motion ensure that $\overline{\boldsymbol{\Pi}}_{C}$ vanishes for arbitrary variations $\delta C$ about a classical solution. Hence, the variational principle associated with $L_{\ddagger}$ is qualitatively similar to the one associated with the original action $L$. Both variational principles feature fixation only of $X$ and the metric data $\overline{\boldsymbol{N}}$ on the $\overline{\mathcal{T}}$ boundary. [ᄆ With regard to the boundary data fixed on $\Sigma^{\prime}$ and $\Sigma^{\prime \prime}$, these two variational principles do, of course, differ. This difference stems from the different choice of $\Sigma$ canonical variables used for each. एण Having established that $L_{\ddagger}$ possesses the correct variational principle, it is now a trivial matter to eliminate the constraints in order to find, in full detail,

$$
\begin{aligned}
\left.L_{\ddagger}\right|^{\text {red }}= & \int_{t^{\prime}}^{t^{\prime \prime}} \mathrm{d} t \mathbf{P}_{\mathbf{C}} \dot{\mathbf{C}}+\int_{\overline{\mathcal{T}}} \mathrm{d} t\left[\sqrt{2 \exp (-\alpha X) \overline{\mathbf{N}}^{2}(\mathbf{C}-W)+(\dot{X})^{2}}\right. \\
& \left.-\frac{1}{2} \kappa \dot{X} \log \left|\frac{\sqrt{2 \exp (-\alpha X) \overline{\mathbf{N}}^{2}(\mathbf{C}-W)+(\dot{X})^{2}}+\dot{X}}{\sqrt{2 \exp (-\alpha X) \overline{\boldsymbol{N}}^{2}(\mathbf{C}-W)+(\dot{X})^{2}}-\dot{X}}\right|\right]
\end{aligned}
$$

\footnotetext{
${ }^{19}$ As is well-known, the boundary lapse is intimately related to the inverse Hawking temperature 42 (blue-shifted from infinity). Therefore, with these quantities fixed at the boundary, our action is closely related to the so-called thermodynamical action appropriate for examining black holes in the canonical ensemble. 21] This consideration further motivates retention of these quantities as fixed data on the $\overline{\mathcal{T}}$ boundary.

${ }^{20}$ With regard to the aforementioned thermodynamical scenario, note that such a difference is irrelevant. In such a scenario, the initial $\Sigma^{\prime}$ and final $\Sigma^{\prime \prime}$ spacelike slices are typically identified (leaving no temporal boundary), as inverse temperature corresponds to periodic imaginary time.
} 
as the promised reduced action. In the reduced action the boundary lapse $\overline{\mathbf{N}}$ is a positive independent parameter. This is the reduced action corresponding to a general ECM for our bounded patch $\mathcal{M}$.

\section{Rest-frame energy}

Let us now derive an expression for the rest-frame energy which is applicable to those GDTs which have an asymptotically flat black-hole solution and a flat-space "linear dilaton" vacuum. Namely, those models within the physical class of $\S$ IV. Again, we continue to assume the vacuum case, although all we really need to require is that matter, if present, has support on a compact set which lies within the outer boundary $\overline{\mathcal{T}}_{o}$. The scenario we study now has been outlined in $\S$ IV. Again, without loss of generality, we set $\alpha=0$. First, performance of the conformal transformation of $\S$ I.C in the action $L_{\ddagger}$ amounts to a simply re-definition,

$$
\tilde{L}_{\ddagger}=\int_{\mathcal{M}} \mathrm{d}^{2} x\left(\mathrm{P}_{C} \dot{C}+\mathrm{P}_{X} \dot{X}+\mathrm{P}_{\xi} \dot{\xi}-N^{C} C^{\prime}-N^{X} \mathrm{P}_{X}-N^{\xi} \mathrm{P}_{\xi}\right)-\int_{\overline{\mathcal{T}}} \mathrm{d} t(\kappa \boldsymbol{\eta} \dot{X}+\tilde{\overline{\boldsymbol{N}}} \tilde{\overline{\boldsymbol{E}}}),
$$

with

$$
\begin{aligned}
\tilde{\overline{\boldsymbol{N}}}^{2} & =\exp (-2 \varphi)\left[2 \kappa^{-2}(C-W)\left(N^{C}\right)^{2}-\frac{1}{2}(C-W)^{-1}\left(N^{X}\right)^{2}\right] \\
\tilde{\overline{\boldsymbol{E}}} & =-\kappa \sqrt{2 \exp (2 \varphi)(C-W)+(\dot{X} / \tilde{\overline{\boldsymbol{N}}})^{2}} \\
\boldsymbol{\eta} & =-\frac{1}{2} \log \left|\frac{\tilde{\overline{\mathbf{N}}} \tilde{\overline{\boldsymbol{E}}}+\kappa \dot{X}}{\tilde{\overline{\mathbf{N}}} \tilde{\overline{\boldsymbol{E}}}-\kappa \dot{X}}\right| .
\end{aligned}
$$

The EOMs associated with $\tilde{L}_{\ddagger}$ are the same ones (6.39) as before; and, moreover, the variational principle associated with $\tilde{L}_{\ddagger}$ only features fixation of $X$ and $\tilde{\bar{N}}$ on the $\overline{\mathcal{T}}$ boundary (and is identical in important respects to the one associated with $L_{\ddagger}$ ).

Next, we shall assume that the variational set of histories associated with the action $\tilde{L}_{\ddagger}$ is determined by a fixed value of $X$ on the $\overline{\mathcal{T}}$ boundary, and so $\dot{X}=0$. This in turn implies that on-shell the quasilocal momentum $\tilde{\bar{J}}=\tilde{\overline{\boldsymbol{J}}}=0$, which is why we are using the term "rest frame." Furthermore, this assumption implies that the $\overline{\mathcal{T}}$ boundary is generated by the flow of the Killing field $k^{\mu}$, or, in other words, that the frames $\left(\tilde{\bar{u}}^{\mu}, \tilde{\bar{n}}^{\mu}\right)$ and $\left(\tilde{\hat{u}}^{\mu}, \tilde{\hat{n}}^{\mu}\right)$ have been identified at the $\overline{\mathcal{T}}$ boundary. This rest-frame condition may be achieved precisely because we are working with an action principle which features fixation of $X$ as boundary data. The action now takes the form

$$
\begin{aligned}
\tilde{L}_{\ddagger}= & \int_{\mathcal{M}} \mathrm{d}^{2} x\left(\mathrm{P}_{C} \dot{C}+\mathrm{P}_{X} \dot{X}+\mathrm{P}_{\xi} \dot{\xi}-N^{C} C^{\prime}-N^{X} \mathrm{P}_{X}-N^{\xi} \mathrm{P}_{\xi}\right) \\
& \int_{\overline{\mathcal{T}}} \mathrm{d} t\left[2 \kappa \lambda^{-1} \tilde{\overline{\mathbf{N}}}|W| \sqrt{1+C|W|^{-1}}\right],
\end{aligned}
$$

where we have chosen $\S$ IV's conformal transformation and assumed those conditions relevant for the class of physical models, $W=-|W|$ and $\lim _{X \rightarrow \infty}|W|=\infty$. 
We subtract from the action a reference term [13][cf. Eq. (1.1)]

$$
\left.\tilde{L}_{\ddagger}\right|^{0}:=\int_{\overline{\mathcal{T}}} \mathrm{d} t\left[2 \kappa \lambda^{-1} \tilde{\bar{N}}|W|\right] \text {. }
$$

Notice that the reference term is a functional only of boundary data which is fixed in the variational principle. Physically, we may interpret $\left.\tilde{L}_{\ddagger}\right|^{0}$ as the action $\tilde{L}_{\ddagger}$ evaluated on the linear dilaton vacuum of $\S$ IV. More precisely, we consider the isometric embedding of the $\overline{\mathcal{T}}$ boundary in flat Minkowski spacetime as the (disjoint) union of inner an outer lines of constant $r=\lambda^{-1} \tilde{X}_{0}$ [cf. Eq. (4.6)]. Here $\tilde{X}_{0}$ is determined by the fixed value of $X$ on either $\overline{\mathcal{T}}_{i}$ or $\overline{\mathcal{T}}_{o}$, as in general $\tilde{X}=\tilde{X}(X)$. One should re-scale the inertial time coordinate in $\left(\mathrm{d} \tilde{s}_{0}\right)^{2}$ accordingly, in order that, with respect to the rescaled time, $-\tilde{\overline{\mathbf{N}}}^{2}$ is the induced metric on the these timelike lines in Minkowski spacetime. Since one is always free to add functionals of the fixed boundary data to the action without affecting the variational principle, we are assured of the fact that the variational principle associated with the new referenced action,

$$
\begin{aligned}
\left.\tilde{L}_{\ddagger}\right|^{\text {ref }}:=\tilde{L}_{\ddagger}-\left.\tilde{L}_{\ddagger}\right|^{0}= & \int_{\mathcal{M}} \mathrm{d}^{2} x\left(\mathrm{P}_{C} \dot{C}+\mathrm{P}_{X} \dot{X}+\mathrm{P}_{\xi} \dot{\xi}-N^{C} C^{\prime}-N^{X} \mathrm{P}_{X}-N^{\xi} \mathrm{P}_{\xi}\right) \\
& -\int_{\overline{\mathcal{T}}} \mathrm{d} t\left[2 \kappa \lambda^{-1} \tilde{\bar{N}}|W|\left(1-\sqrt{1+C|W|^{-1}}\right)\right]
\end{aligned}
$$

is the same as before. As with (6.38) above, elimination of the constraints in (6.47) in order to find the corresponding reduced action is a trivial matter.

The (outer boundary-point) referenced quasilocal energy we "read off" from the reduced form of the canonical action (6.47) is the following one:

$$
\left.\tilde{\tilde{\boldsymbol{E}}}\right|^{\text {ref }}=2 \kappa \lambda^{-1}|W|\left[1-\sqrt{1+\mathbf{C}|W|^{-1}}\right]
$$

This expression for the rest-frame QLE agrees with the result obtained in Ref. [35] by Barvinsky and Kunstatter directly from the Hamiltonian for a GDT (taking $C^{\prime}$ in place of the usual Hamiltonian constraint) via a Regge-Teitleboim-type argument [43]. That is, agreement is obtained, provided that one chooses a unit boundary lapse for the expression in that reference (as is appropriate, since the QLE is the Hamiltonian value corresponding to a pure time translation [13]). The $X \rightarrow \infty$ limit of $\left.\tilde{\overline{\boldsymbol{E}}}\right|^{\text {ref }}$ defines the analog of the ADM mass (asymptotic rest-frame energy) for the models we consider [13,20]. We find

$$
\left.M_{A D M} \equiv \lim _{X \rightarrow \infty} \tilde{\tilde{\boldsymbol{E}}}\right|^{\text {ref }}=-\kappa \lambda^{-1} \mathbf{C} .
$$

With the SSGR choice (1.9a) for $\kappa, M_{A D M}$ is the Schwarzschild mass parameter $M_{S}$ (as expressed in terms of $\mathbf{C}=C$ as given in $\S$ III and IV). More generally, taking $\kappa=1$ (as is perhaps more appropriate for a $2 \mathrm{~d}$ model), we obtain $M_{A D M}=-\lambda^{-1} \mathbf{C}$ (mass dimension 1 in $2 \mathrm{~d}$ ). Finally, we mention that the quasilocal approach employed here may also be used to define total gravitational energy-momentum at null infinity. 44,45] 


\section{CONCLUSIONS}

Our main results in this paper concern the important role played by boundary conditions in the passage -via conformal transformation- between Einstein-Cartan models (ECMs, described by a first-order zweibein action) and generalized dilaton theories (GDTs, described by a metric action). Once again, we have been motivated to study this equivalence because, although it is GDT which more nearly mimics physical features of $4 \mathrm{~d}$ spherically symmetric general relativity (SSGR) and thus provides more interesting 2d models of gravity, it is far more tractable mathematically to work within the first-order framework associated with ECMs. As shown in $\S \mathrm{I}$, if the intent is to perform such a conformal re-definition of field variables in the first-order action for an ECM, then a boundary term must be included in the definition of the action, or else the action's associated variational principle is not preserved under the the conformal transformation. Moreover, while at first sight it appears that the addition of such a boundary term destroys the zweibein gauge-invariance of the theory at the boundary $\partial \mathcal{M}$ of the spacetime patch $\mathcal{M}$, we have explicitly demonstrated in $\S \mathrm{V}$ that this need not be true. It is quite notable that by imposing the dual requirements of (i) conformally preserved boundary conditions and (ii) complete zweibein-gauge invariance, we have arrived at set of canonical variables, namely, the $\Sigma$-adapted canonical variables ( $\Sigma$ CVs) (5.12), which appear to be new in the literature. Although strictly zweibein variables, the $\Sigma$ CVs are intimately related to the standard canonical ADM metric variables, and therefore, they particularly elucidate the relationship between zweibein ECMs and metric GDTs. Moreover, as we discuss below, the $\Sigma$ CVs also play an important part in the overall picture of the inter-relationships between several author's approaches to $1+1$ canonical gravity.

We have also throughly explored the notion of gravitational energy and momentum in $1+1$ gravity. Besides being of interest in its own right, the canonical quasilocal energymomentum (here derived via a Hamilton-Jacobi-type argument due to Brown and York [13) serves as a stepping stone for introducing the rapidity in $\S \mathrm{V}$.B, thus leading to the rapidity canonical variables (RCVs) in $\S$ VI.B. With the $\Sigma$ CVs and RCVs in mind, let us return to the various canonical transformations examined in the last section. A "flow chart" in Fig. 2 depicts the inter-relationships between the various canonical variables we have introduced. At the top of the chart are the ECVs. The chain of transformations labeled 1, 2 , and 3 correspond to passage from a zweibein ECM to a metric GDT. As we have noted in $\S$ VI.A, one could interchange the order of steps 2 and 3, i. e. the canonical transformation corresponding to the conformal rescaling can be performed prior to elimination of the gauge pair $\left\{P_{\rho}, \rho\right\}$. Notice that in the 1-2-3 chain, no generating functionals are required. This further underscores the significance of the $\Sigma$ CVs at the top of the chart and why we have called them " $\Sigma$-adapted." Steps I and A depict the canonical transformation to the RCVs (6.12). Essentially the same generating functional, $\Xi$ given in Eq. (6.16), is required for both steps. From the RCVs, one may easily pass to the "true degrees of freedom" via the chain labeled by B-C. Not depicted in our flow chart are the Possion-sigma-model canonical variables [cf. the discussion immediately preceding Eq. (6.11)] of Klösch and Strobl [6], although as our main discussion indicates they lie half-way between the $\Sigma$ cvs and the RCVs (in the middle of the A transformation). The canonical transformations II and III, made after elimination of the Lorentz constraint, are quite closely related to the canonical

transformation made in I (or in A). In fact, the generating functionals $\Xi^{\prime}$ and $\tilde{\Xi}^{\prime}$ for these 
steps are defined from $\Xi$ by elimination of the Lorentz constraint, ${ }^{\top}$ i. e. $\Xi^{\prime}=\tilde{\Xi}^{\prime}=\left.\Xi\right|_{G=0}$. Clearly, $\Xi^{\prime}$ and $\tilde{\Xi}^{\prime}$, although equal to each other, are expressed in terms of different canonical variables. In fact, one finds

$$
\begin{aligned}
\left.\Xi^{\prime}\right|_{\Sigma} & :=-\int_{\Sigma} \mathrm{d} r\left[\Lambda P_{\Lambda}+\frac{1}{2} X^{\prime} \log \left|\frac{X^{\prime}+\Lambda P_{\Lambda}}{X^{\prime}-\Lambda P_{\Lambda}}\right|\right] \\
& =-\int_{\Sigma} \mathrm{d} r\left[\tilde{\Lambda} \mathcal{P}_{\tilde{\Lambda}}+\frac{1}{2} X^{\prime} \log \left|\frac{X^{\prime}+\tilde{\Lambda} \mathcal{P}_{\tilde{\Lambda}}}{X^{\prime}-\tilde{\Lambda} \mathcal{P}_{\tilde{\Lambda}}}\right|\right]=:\left.\tilde{\Xi}^{\prime}\right|_{\Sigma}
\end{aligned}
$$

For the case of vacuum SSGR, the generating functional $\tilde{\Xi}^{\prime}$ is precisely the one considered by Kuchař in his thorough paper on the canonical geometrodynamics of SBHs 清. This generating functional also plays an important role in Louko and Whiting's examination (employing Kuchař's approach) of the Hamiltonian thermodynamics of SBHs [22]. For vacuum 2dDG it corresponds to the generating functional considered in Ref. [20]. At the level of full GDT, it is precisely such generating functionals which correspond to the transformations studied by Kunstatter and Barvinsky [35].

Finally, let us discuss what we have not done in this paper. First, although we have striven to point out precisely where and why the inclusion of matter does not affect our results, we have not taken matter explicitly into account. It would be interesting to examine matter-induced gravitational collapse in GDT along the same lines as carried out in SSGR by Guven and Ó Murchadha [30], especially as mathematically their formalism is quite similar to the one studied here [cf. the footnote just before Eq. (5.15)]. The known existence of critical behavior for gravitational collapse in SSGR would seem to indicate that there are, in fact, a wide class of models where such criticality could be examined (here non-minimally coupled matter would be a prerequisite). For treatments of the coupled matter-gravity CGHS model [11], Refs. [33,46] should be consulted. Second, we have bypassed the stubborn problem of either (i) finding good canonical variables ${ }^{2 z}$ which do not break down on horizons or, at the very least, (ii) developing a precise understanding of how to use known "targetspace" canonical variables (such as our RCVs) when a spacelike slice cuts completely across a horizon in a general way. As mentioned, for SSGR Kuchař has studied in detail option (ii) [4], and his treatment is the most serious attempt in this direction known to us. However, in our opinion this issue is quite deserving of further research.

\footnotetext{
${ }^{21}$ Note that neither $\Xi^{\prime}$ nor $\tilde{\Xi}^{\prime}$ are the same as the alternative generating functional $\Upsilon$ considered in $\S$ VI. However, it is true that $\Xi^{\prime}=\tilde{\Xi}^{\prime}=\left.\Upsilon\right|_{G=0}$. Of course, $\Upsilon \neq\left.\Xi\right|_{G=0}$.

${ }^{22}$ Of course, depending on the temporal foliation, the plain ADM variables certainly may be wellbehaved across a horizon; but we seek variables which simplify (preferably drastically) the constraints as well. The ADM variables do not yield such simplification.
} 


\section{ACKNOWLEDGMENTS}

For helpful discussions and insight we thank H. Balasin, H. Liebl, N. Ò Murchadha, and, particularly, both T. Klösch and T. Strobl. This research has been supported by the "Fonds zur Förderung der wissenschaftlichen Forschung" in Austria (FWF project 10.221PHY). S. R. Lau also gratefully acknowledges support as a Lise Meitner Fellow of the FWF (Project M-00182-PHY). 


\section{REFERENCES}

[1] For a sample of relevant literature see the first citation of Ref. 13.

[2] P. Thomi, B. Isaak, and P. Hájíček, Phys. Rev. D30, 1168 (1984).

[3] T. Thiemann and H. A. Kastrup, Nucl. Phys. B399, 211 (1993); T. Thiemann and H. A. Kastrup, Nucl. Phys. B399, 221 (1993); H. A. Kastrup and T. Thiemann, Nucl. Phys. B425, 665 (1994); T. Thiemann, Nucl. Phys. B436, 681 (1995).

[4] K. V. Kuchař, Phys. Rev. D50, 3961 (1994).

[5] T. Strobl, Phys. Rev. D50, 7346 (1994); P. Schaller and T. Strobl in Lecture Notes in Physics 436, edited by A. Alekseev et al (Springer 1994); P. Schaller and T. Strobl, Mod. Phys. Lett. A9, 3129 (1994); T. Strobl, Poisson-structure Induced Field Theory and Models of $1+1$ Dimensional Gravity, TU Wien Ph.D. dissertation (Vienna, June 1994); P. Schaller and T. Strobl in the Proceedings of the International Workshop on "Finite Dimensional Integrable Systems," edited by A. N. Sissakian and G. S. Pogosyan (Dubna 1995); P. Schaller and T. Strobl in Lecture Notes in Physics 469, edited by H. Grosse and L. Pittner (Springer 1996).

[6] T. Klösch and T. Strobl, Class. Quantum Grav. 13, 965 (1996); Class. Quantum Grav. 13, 2395 (1996); Classical and Quantum Gravity in 1+1 Dimensions: Part III, hep-th/9607226.

[7] W. Kummer and P. Widerin, Phys. Rev. D52, 6965 (1995).

[8] M. O. Katanaev, W. Kummer and H. Liebl, Phys. Rev. D53, 5609 (1996).

[9] G. Mandal, A. Sengupta and S. R. Wadia, Mod. Phys. Lett. A6, 1685 (1991), 1685 (preprint IASSNS-HEP-91/10).

[10] E. Witten, Phys. Rev D44, 314 (1991).

[11] C.G. Callen, S. B. Giddings, J. A. Harvey, and A. Strominger, Phys. Rev. D45, R1005 (1992); A. Strominger, Les Houches lectures on black holes, Lectures presented at the 1994 Les Houches Summer School "Fluctuating Geometries in Statistical Mechanics and Field Theory," gr-qc/9501071.

[12] M. O. Katanaev, W. Kummer and H. Liebl, On the Completeness of the Black Holes Singularity in 2d Dilaton Theories, gr-qc/9605004 (to appear in Nucl. Phys. B).

[13] J. D. Brown and J. W. York, Phys. Rev. D47, 1407 (1993).

[14] C. W. Misner and D. W. Sharp, Phys. Rev. 136 B571 (1964).

[15] S. W. Hawking, J. Math. Phys. 9, 598 (1968); R. Penrose, Proc. Roy. Soc. Lond. A381 53 (1982); A. J. Dougan and L. J. Mason, Phys. Rev. Lett. 672119 (1991); A. J. Dougan, Class. Quantum Grav. 92461 (1992); S. A. Hayward, Phys. Rev. D49, 831 (1994); Phys. Rev. D53, 1938 (1996).

[16] R. B. Mann, A. Shiekh and L. Tarasov, Nucl. Phys. B341, 134 (1990); D. Banks and M. O'Loughlin, Nucl. Phys. B362, 649 (1991); S. D. Odintsov and I. J. Shapiro, Phys. Lett. B263, 183 (1991), Mod. Phys. Lett. A7, 437 (1992); I. G. Russo and A. A. Tseytlin, Nucl. Phys. B382, 259 (1992); I. V. Volovich, Mod. Phys. Lett. A, 1827 (1992); R. B. Mann, Phys. Rev. D47, 4438 (1993); D. Louis-Martinez, J. Gegenberg, and G. Kunstatter, Phys. Rev. D49, 5227 (1994); J. S. Lemos and P. M. Sa, Phys. Rev. D49, 2897 (1994).

[17] R. Arnowitt, S. Deser, and C. W. Misner, in Gravitation: An Introduction to Current Research, edited by L. Witten (Wiley, New York, 1962). 
[18] G. W. Gibbons and S. W. Hawking, Commun. Math. Phys. 66, 291 (1979).

[19] K. C. K. Chan, J. D. E. Creighton, and R. B. Mann, Conserved masses in GHS Einstein and string black holes and consistent thermodynamics, gr-qc/9604055.

[20] S. R. Lau, Class. Quantum Grav. 13, 1541 (1996).

[21] J. W. York, Phys. Rev. D33, 2092 (1986); H. W. Braden, B. F. Whiting, and J. W. York, Phys. Rev. D36, 3614 (1987); B. F. Whiting and J. W. York, Phys. Rev. Lett. 61, 1336 (1988); J. W. York, Physica A158, 425 (1989); J. D. Brown, G. L. Comer, E. A. Martinez, J. Melmed, B. F. Whiting, and J. W. York, Class. Quantum Grav. 7, 1433 (1990); H. W. Braden, J. D. Brown, B. F. Whiting, and J. W. York, Phys. Rev. D42, 3376 (1990); J. W. York in Conceptual Problems of Quantum Gravity, edited by A. Ashtekar and J. Stachel (Birkhäuser, Boston, 1991); J. D. Brown, E. A. Martinez, and J. W. York, Phys. Rev. Lett. 66, 2281 (1991); J. D. Brown and J. W. York, Phys. Rev. D47, 1420 (1993); J. D. Brown and J. W. York, The path integral formulation of gravitational thermodynamics, gr-qc/9405024, based on a talk presented by J. D. Brown at the conference The Black Hole 25 Years After (Santiago, Chile 1994).

[22] J. Louko and B. F. Whiting, Phys Rev. D51, 5583 (1995).

[23] G. Hayward and K. Wong, Phys. Rev. D46, 620 (1992); G. Hayward, Phys. Rev. D47, 4778 (1993).

[24] S. R. Lau, Canonical Variables and Quasilocal Energy in General Relativity, University of North Carolina Ph. D. dissertation (Chapel Hill, June 1994); Class. Quantum Grav. 13, 1509 (1996).

[25] W. Kummer, D. J. Schwarz, Nuc. Phys. B382, 171 (1992).

[26] C. W. Misner, K. S. Thorne, and J. A. Wheeler, Gravitation (Freeman, San Francisco, 1973).

[27] M. O. Katanaev and I. V. Volovich, Phys. Lett. 175B 413 (1986); Ann. Phys. 197, 1 (1990).

[28] P. R. Brady, Phys. Rev. D51, 4168 (1995).

[29] P. Schaller and T. Strobl, Class. Quantum Grav. 11, 331 (1994).

[30] J. Guven and N. Ó Murchadha, Phys. Rev. D52, 758 (1995); Phys. Rev. D52, 776 (1995); Constraints in Spherically Symmetric Classical General Relativity: Part III, gr-qc/9504039.

[31] T. Tada and S. Uehara, Phys. Rev. D51, 4259 (1995); Phys. Lett. B 305, 23 (1993).

[32] V. P. Frolov, Phys. Rev. D46, 5383 (1992).

[33] S. A. Hayward, Class. Quantum Grav. 10, 985 (1993).

[34] D. Louis-Martinez, J. Gegenberg, and G. Kunstatter, Phys. Letss. B321, 193 (1994); Phys. Rev. D52 3494 (1995).

[35] A. Barvinsky and G. Kunstatter, Mass Spectrum for Black Holes in Generic 2-D Dilaton Gravity, gr-qc/9607030; Exact Physical Black Hole States in Generic 2-D Dilaton Gravity, hep-th/9606134.

[36] J. W. York, Phys. Rev. Lett. 28, 1082 (1972); J. W. York, Found. Phys. 16, 249 (1986).

[37] D. Brill and G. Hayward, Phys. Rev. D50, 4914 (1994).

[38] E. Malec and N. Ó Murchadha, Phys. Rev. 50, R6033 (1994).

[39] M. Varadarajan, Phys. Rev. D52, 7080 (1995).

[40] S. Bose, J. Louko, L. Parker, and Y. Peleg, Phys. Rev. D53, 570 (1996); J. Louko and S. N. Winters-Hilt, Hamiltonian thermodynamics of the Reissner-Nordström-anti- 
de Sitter black hole, gr-qc/9602003.

[41] W. Fischler, D. Morgen, and J. Polchinsky, Phys. Rev. D42, 4042 (1990).

[42] S. W. Hawking, Commun. Math. Phys. 43, 199 (1975).

[43] T. Regge and C. Teitleboim, Ann. Phys. (N. Y. ) 88, 286 (1974).

[44] H. J. W. Müller-Kirsten, J.-G. Zhou, Y.-G. Miao, and J.-Q.Liang, Phys. Lett. B362, $51(1995)$.

[45] J. D. Brown, S. R. Lau, and J. W. York, Energy of Isolated Systems at Retarded Times as the Null Limit of Quasilocal Energy, gr-qc/9609057 (to appear in Phys. Rev. D).

[46] K. V. Kuchař, J. D. Romano, and M. Varadarajan, Dirac Quantization of a Dilatonic Model of Gravitational Collapse, gr-qc/9608011 (to appear in Phys. Rev. D). 


\section{FIGURES}

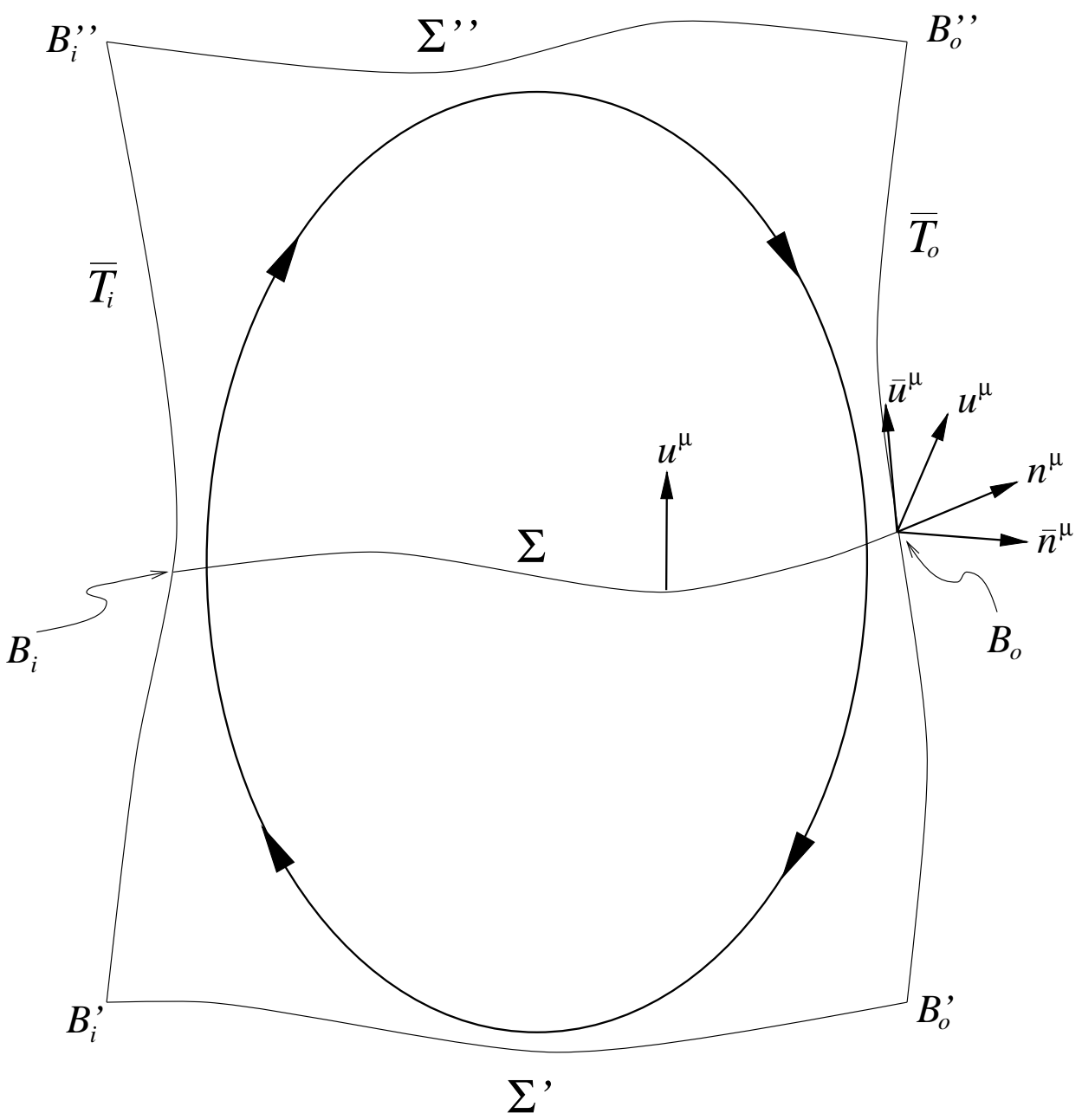

FIG. 1. Spacetime Patch $\mathcal{M}$

The figure depicts the geometry of our spacetime patch $\mathcal{M}$. Here $\Sigma$ represents a generic 1dimensional spacelike slice with a boundary consisting of two points, $B_{i}$ and $B_{o}$. At the outer boundary point $B_{o}$ the time-gauge zweibein $\left(u^{\mu}, n^{\mu}\right)$ and the radial-gauge zweibein $\left(\bar{u}^{\mu}, \bar{n}^{\mu}\right)$ are drawn. Note that $n^{\mu}$ points tangentially to $\Sigma$ and is the outward-pointing normal of $B_{o}$ as embedded in $\Sigma$. The vector $\bar{u}^{\mu}$ points tangentially to the 1 -dimensional timelike curve $\overline{\mathcal{T}}_{o}$ (one element of the full boundary $\partial \mathcal{M}$ ) and is the future-pointing normal of $B_{o}$ as embedded in $\overline{\mathcal{T}}_{o}$. All the elements of $\partial \mathcal{M}$, namely, $\Sigma^{\prime}, \Sigma^{\prime \prime}, \overline{\mathcal{T}}_{i}$, and $\overline{\mathcal{T}}_{o}$, are depicted. Notice that $\eta=\sinh ^{-1}\left(-u_{\mu} \bar{u}^{\mu}\right)$ need not vanish at the outer boundary point $B_{o}$. For that matter, the boost parameter $\eta$ need not vanish at the corner points: $B_{i}^{\prime}, B_{i}^{\prime \prime}, B_{o}^{\prime}$, and $B_{o}^{\prime \prime}$. The heavy ellipse inscribed within $\mathcal{M}$ illustrates our convention for the orientation of the boundary $\partial \mathcal{M}$ as embedded in $\mathcal{M}$. The chosen "clockwise" orientation determines the integration conventions (5.2). 


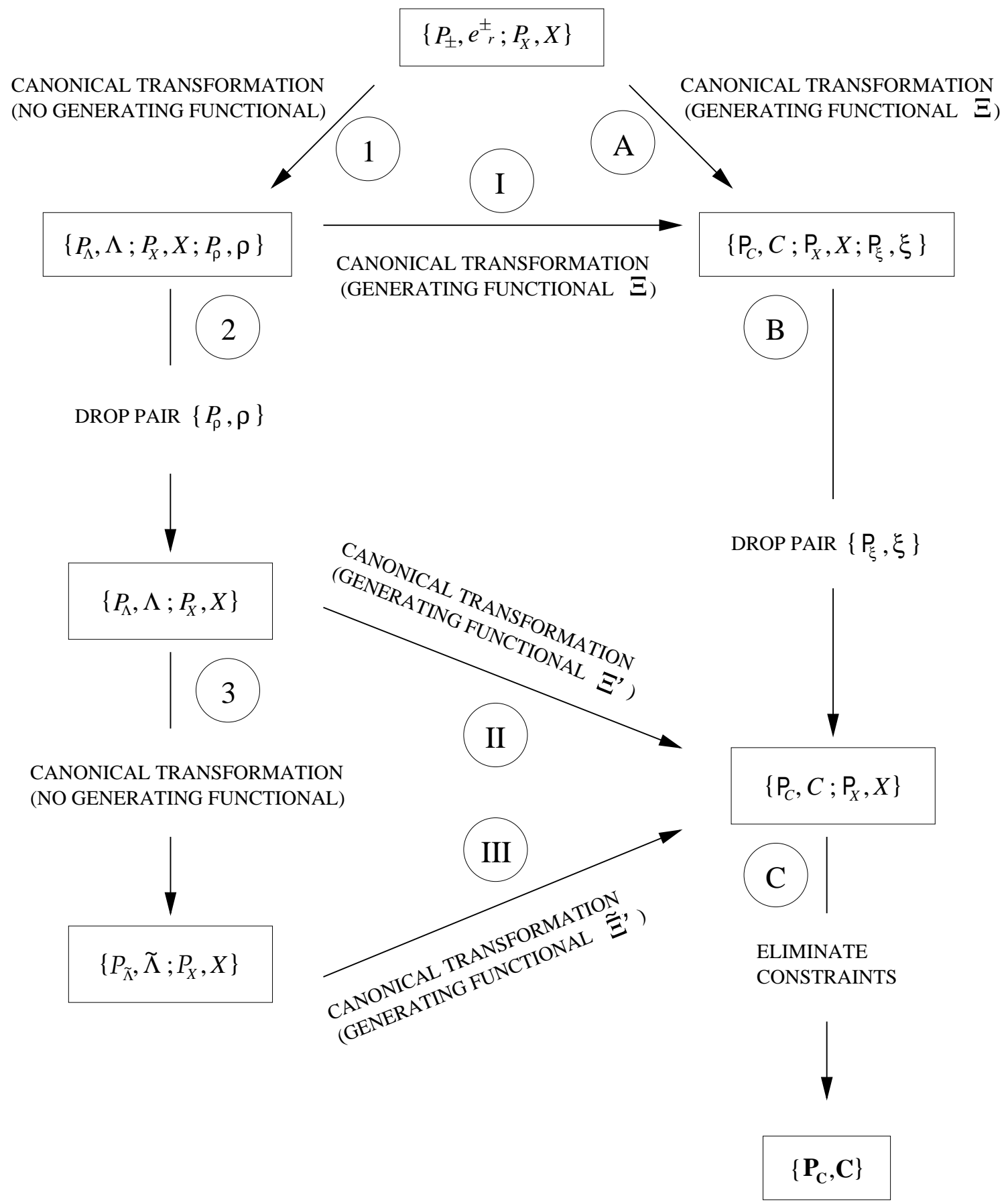

FIG. 2. Canonical Variables and Transformations from $\S$ VI

The various canonical variables and canonical transformations depicted in this flow chart are described in full in $\S$ VII, the concluding section. 